\title{
Unmet care needs of advanced cancer patients and their informal caregivers: a systematic review
}

Tao Wang ${ }^{1}$, Alex Molassiotis ${ }^{1 *}$, Betty Pui Man Chung ${ }^{1}$ and Jing-Yu Tan ${ }^{1,2}$

\begin{abstract}
Background: This systematic review aimed to identify the unmet care needs and their associated variables in patients with advanced cancer and informal caregivers, alongside summarizing the tools used for needs assessment.

Methods: Ten electronic databases were searched systematically from inception of each database to December 2016 to determine eligible studies. Studies that considered the unmet care needs of either adult patients with advanced cancer or informal caregivers, regardless of the study design, were included. The Mixed Methods Appraisal Tool was utilized for quality appraisal of the included studies. Content analysis was used to identify unmet needs, and descriptive analysis was adopted to synthesize other outcomes.
\end{abstract}

Results: Fifty studies were included, and their methodological quality was generally robust. The prevalence of unmet needs varied across studies. Twelve unmet need domains were identified in patients with advanced cancer, and seven among informal caregivers. The three most commonly reported domains for patients were psychological, physical, and healthcare service and information. The most prominent unmet items of these domains were emotional support (10.1-84.4\%), fatigue (18-76.3\%), and "being informed about benefits and side-effects of treatment" (4-66.7\%). The most commonly identified unmet needs for informal caregivers were information needs, including illness and treatment information (26-100\%) and care-related information (21-100\%). Unmet needs of patients with advanced cancer were associated with their physical symptoms, anxiety, and quality of life. The most commonly used instruments for needs assessment among patients with advanced cancer were the Supportive Care Needs Survey $(N=8)$ and Problems and Needs in Palliative Care questionnaire $(N=5)$. The majority of the included studies investigated unmet needs from the perspectives of either patients or caregivers with a cross-sectional study design using single time-point assessments. Moreover, significant heterogeneity, including differences in study contexts, assessment methods, instruments for measurement, need classifications, and reporting methods, were identified across studies.

Conclusion: Both advanced cancer patients and informal caregivers reported a wide range of context-bound unmet needs. Examining their unmet needs on the basis of viewing patients and their informal caregivers as a whole unit will be highly optimal. Unmet care needs should be comprehensively evaluated from the perspectives of all stakeholders and interpreted by using rigorously designed mixed methods research and longitudinal studies within a given context.

\footnotetext{
* Correspondence: alex.molasiotis@polyu.edu.hk

${ }^{1}$ School of Nursing, The Hong Kong Polytechnic University, Hung Hom, Hong

Kong

Full list of author information is available at the end of the article
}

(c) The Author(s). 2018 Open Access This article is distributed under the terms of the Creative Commons Attribution 4.0 International License (http://creativecommons.org/licenses/by/4.0/), which permits unrestricted use, distribution, and reproduction in any medium, provided you give appropriate credit to the original author(s) and the source, provide a link to the Creative Commons license, and indicate if changes were made. The Creative Commons Public Domain Dedication waiver (http://creativecommons.org/publicdomain/zero/1.0/) applies to the data made available in this article, unless otherwise stated. 


\section{Background}

According to the World Health Organization (WHO), more than 15 million people will be diagnosed with cancer by 2020 [1]. With the advances in cancer treatments, the illness trajectory and prognosis of cancer have changed, and patients diagnosed with advanced cancer can live for a relatively long period [2, 3]. However, lengthy cancer experience and anticancer treatments make patients suffer from a wide range of problems, such as physical, psychological, emotional, and practical issues [4]. Cancer-related symptoms and patients' experiences during cancer treatment vary across different cancer stages, and patients at advanced stage commonly experience different symptoms from those with early-stage cancer $[5,6]$. Such 'chronic and uncertain' conditions pose a challenge to not only the cancer services but also to their informal caregivers [7]. Informal caregivers commonly take care of their loved ones for a long period [8]. The long-term caregiving process is physically and psychologically challenging, particularly when taking care of patients with advanced cancer [9]. Many informal caregivers, including those who do not regard caregiving as a burden, suffer from a wide range of problems, such as sleep disturbance, anxiety, depression, and practical and financial difficulties $[10,11]$. Informal caregivers are usually regarded as fellow sufferers alongside patients [12]. Unmet needs of patients can increase the level of caregiver burden [13]. In turn, caregivers' problems are closely linked with patients' well-being [14], and unsolved problems or unmet needs of caregivers will not only decrease their own quality of life [15] but also affect the patients' health outcomes negatively [15]. Informal caregivers and patients with advanced cancer are considered a whole unit in fighting the illness [10].

High-quality and patient-and-family-centered care is needed to address the problems of both the advanced cancer patients and their informal caregivers, including symptom and side effect management, as well as emotional, psychosocial, and spiritual support. All these aspects of support are typically categorized under palliative care [16]. Mismatched healthcare that is inconsistent with patients and caregivers' needs can increase healthcare expenditure and lead to harmful effects [17]. Therefore, the unmet care needs of patients and informal caregivers should be comprehensively assessed prior to designing and providing tailored palliative care services [18, 19]. Care needs are defined as "the requirement of some action or resource in care that is necessary, desirable, or useful to attain optimal well-being" (Foot, 1996, as cited in Sanson-Fisher, et al., 2000, p.227) [20]. Unmet needs assessment is designed to identify how well and how much their needs have been satisfied or not [21]. An early review [17] summarized the instruments for needs assessment; however, a majority of these instruments have been designed for general patients with cancer (e.g., Supportive Care Needs Survey, SCNS [17]). After the publication of that review, several tools that were specifically designed for advanced cancer patients (e.g., Needs Assessment of Advanced Cancer Patients, NA-ACP [22]) have been developed and used.

An early systematic review [21] published in 2009 analyzed the unmet needs of patients with advanced cancer with nine included studies. Another systematic review [7] with 23 studies reported eight unmet need domains. These two systematic reviews only focused on patients, with limited literature searches in only four databases. Meanwhile, the inclusion criteria were relatively ambiguous in the second review because studies with mixed samples (patients at different cancer stages) were included; moreover, the definition of advanced cancer was not presented [7]. Moreover, neither of the two reviews summarized and reported detailed information regarding the needs assessment tools used, which is important information to allow readers to appreciate the quality and reliability of study results. Furthermore, to date, no systematic review has been conducted to explore the unmet needs of informal caregivers of patients with advanced cancer. Therefore, the current systematic review was carried out to update evidence from previous reviews and provide a more comprehensive picture regarding the unmet needs among patients with advanced cancer and informal caregivers. An intensive literature search was performed on 10 electronic databases, and the inclusion criteria were more specific for advanced cancer diagnosis than those of the previous reviews. This current systematic review also included informal caregivers on the basis of the following concepts: fellow sufferers [12], a whole unit [10], and patient-and-family-centered care that is emphasized by the WHO [16]. Specific objectives of this review included: (1) to identify the unmet care needs and their associated factors in patients with advanced cancer and their informal caregivers, and (2) to summarize needs assessment tools that were used in the included studies.

\section{Methods \\ Search strategies}

With consideration of the language expertise of the review authors, English and Chinese databases were included. Ten databases, including PubMed, Cumulative Index to Nursing and Allied Health Literature (CINAHL), EMBase, Cochrane Central Register of Controlled Trials (CENTRAL), PsycINFO, Web of Science, Wan Fang Data, China National Knowledge Infrastructure (CNKI), Chongqing VIP (CQVIP), and Chinese Biomedical Literature Database (CBM), were searched systematically from inception of each database to December 2016. Restrictions regarding study design were not set. The used $\mathrm{MeSH}$ terms, key words, and free words included needs assessment, assessment of healthcare needs, unmet needs, 
neoplasms, advanced cancer, terminal cancer, metastatic cancer, and the forth. Manual searches were also conducted by examining the reference lists of the included studies. Three representative search strategies of this systematic review are listed in Table 1.

\section{Study identification and data extraction}

Duplications were identified and eliminated through a reference management software (NoteExpress). Titles and abstracts of the remaining studies were screened independently by two review authors (WT and TJY), and full text of potentially eligible studies were subsequently located for further screening. Studies satisfying the following inclusion criteria were included: (1) studies that included either adult ( $\geq 18$ years old) patients with advanced cancer $^{1}$ or adult informal caregivers of patients with advanced cancer; (2) studies that reported data in terms of unmet care needs ${ }^{2}$ or concerns that are directly linked to the unmet care needs of patients with advanced cancer and/or their informal caregivers, regardless of the study design; and (3) accessible full texts were published in peer-reviewed journals. Exclusion criteria were: (1) studies with mixed sample of patients with cancer at any cancer stage (except those patients with advanced cancer who were analyzed separately); (2) studies solely focusing on quality of life [21], satisfaction with healthcare services, care service utilization, or presence of symptoms/problems; (3) studies focusing on instrument development, translation, or evaluation; and (4) conference articles with only abstracts, editorial comments, guidelines, policies, or treatment recommendations. Data were extracted by two independent review authors. These data included information regarding the first author of the study, year of publication, country of origin, research setting, research design, sampling approach, sample size, need assessment methods (interview or other instruments), prevalence of unmet needs, and related factors for unmet needs. Any disagreement was settled and discussed by the two other review authors (CPM and AM).

\section{Methodological quality appraisal}

The methodological quality of included studies was assessed by two review authors (WT and TJY) independently with the Mixed Methods Appraisal Tool (MMAT) [25]. This tool is highly efficient; it takes approximately 14 min to evaluate one study [25] with robust consistency among reviewers (intraclass correlation = 0.72 [25]); MMAT is specifically designed to assess the quality of either quantitative or qualitative studies. Four different quality criteria for qualitative studies and different types of quantitative studies, including randomized control trials, quantitative nonrandomized trials, and quantitative descriptive studies, were used [25]. Each criterion was graded as 0 (unmet) or 1 (meet), and the
Table 1 Selected Search Strategies

PubMed

\#1 Search (((("needs assessment"[MeSH Terms]) OR "needs assessment"[Title/Abstract]) OR "assessment of healthcare needs"[Title/Abstract]) OR "assessment of health care needs" [Title/ Abstract]) OR "unmet needs"[Title/Abstract]

\#2 Search (((c((“"palliative care"[MeSH Terms]) OR "palliative medicine"[MeSH Terms]) OR "hospice care"[MeSH Terms]) OR "supportive care"[Title/Abstract]) OR "palliative nursing"[Title/ Abstract]) OR "palliative care nursing"[Title/Abstract]) OR "terminal care"[Title/Abstract]) OR "hospice nursing care"[Title/Abstract]

\#3 Search ((()(“neoplasms"[MeSH Terms]) OR "advanced cancer" $[$ Title/ Abstract]) OR "terminal cancer"[Title/Abstract]) OR "metastatic cancer"[Title/Abstract]) OR "tumor"[Title/Abstract]) OR "cancer"[Title/ Abstract]

\#4 \#1 AND \#2 AND \#3

CINAHL

\#1 TI needs assessment OR TI assessment of healthcare needs OR TI assessment of health care needs OR TI unmet needs

\#2 AB needs assessment OR AB assessment of healthcare needs $O R$ $A B$ assessment of health care needs $O R A B$ unmet needs

\#3 $A B$ palliative care $O R A B$ palliative medicine $O R A B$ hospice care $O R A B$ supportive care $O R A B$ palliative nursing $O R A B$ palliative care nursing $O R A B$ terminal care $O R A B$ hospice nursing

\#4 TI palliative care OR TI palliative medicine OR TI hospice care OR TI supportive care OR TI palliative nursing OR TI palliative care nursing OR TI terminal care OR TI hospice nursing

\#5 TI neoplasms OR TI tumor OR TI cancer OR TI advanced cancer OR $\mathrm{TI}$ terminal cancer OR TI metastatic cancer

\#6 $A B$ neoplasms $O R A B$ tumor $O R A B$ cancer $O R A B$ advanced cancer $O R A B$ terminal cancer $O R A B$ metastatic cancer

\#7 \#1 OR \#2

\#8 \#3 OR \#4

\#9 \#5 OR \#6

\#10 \#7 AND \#8 AND \#9

EMBase

\#1 'needs assessment'/exp

\#2 'needs assessment':ab,ti OR (assessment:ab,ti AND of:ab,ti AND healthcare:ab,ti AND needs:ab,ti) OR (assessment:ab,ti AND of:ab,ti AND health:ab,ti AND care:ab,ti AND needs:ab,ti) OR 'unmet needs':ab,ti

\#3 \#1 OR \#2

\#4 'palliative care':ab,ti OR 'palliative medicine':ab,ti OR 'hospice care':ab,ti OR 'supportive care':ab,ti OR 'palliative nursing':ab,ti OR 'terminal care':ab,ti OR 'hospice nursing':ab,ti

\#5 'palliative nursing'/exp

\#6 \#4 OR \#5

\#7 'advanced cancer'/exp

\#8 'neoplasm'/exp

\#9 'advanced cancer':ab,ti OR (terminal:ab,ti AND cancer:ab,ti) OR (metastatic:ab,ti AND cancer:ab,ti) OR neoplasm:ab,ti OR cancer:ab,ti OR tumor:ab,ti

\#10 \#7 OR \#8 OR \#9

\#11 \#3 AND \#6 AND \#10 
global score of each study was calculated from 0 to 4 ( 0 $=$ no criterion satisfied, $1=$ satisfied one criterion, $2=$ satisfied two criteria, $3=$ satisfied three criteria, and $4=$ satisfied all four criteria). When any disagreement occurred, the review authors conducted a group discussion to reach final agreement.

\section{Data analysis}

Content analysis [26] was used to identify the unmet need domains of patients with advanced cancer and informal caregivers across quantitative and qualitative studies. A priori content categories of patients with advanced cancer were determined on the basis of previous studies; these categories included health system and information, patient care and support, activities of daily living (ADL), physical, psychological, financial, and spiritual [7]. With regards to informal caregivers, five content categories were determined on the basis of a previous review [10]; these categories included cancer care services, informational, psychological, spiritual, and social needs. Data of the included studies were compared, combined, and clustered with respect to those domains for patients and informal caregivers. Terms, such as instrumental and personal care, were included in the
ADL domain because they were frequently mentioned in several North American studies [21]. Summative content analysis was used to identify and extract new categories within content not covered by previous domains. The approach of descriptive analysis was used for the prevalence of unmet needs due to the significant heterogeneity of the included studies [27]. Variables associated with patients and informal caregivers' needs and used instruments were analyzed through descriptive approach.

\section{Results $^{3}$}

\section{Characteristics of included studies}

Among the 4277 potentially eligible studies, 45 studies were included. After screening the reference lists, five other eligible studies were retrieved. Finally, 50 studies $[6,9,28-75]$ (5 published in Chinese and 45 in English language) were included in this review (Fig. 1). The majority of the studies $(43 / 50)$ used quantitative study designs, with 42 surveys (1 longitudinal survey [75] and 41 cross-sectional surveys) and 1 [6] pre-post intervention study (only baseline data were used in this review). The seven other studies [48, 49, 57, 62, 71-73] were qualitative designs with individual in-depth interviews and/or focus group. Among the 50 included studies, 33 studies investigated the unmet needs of

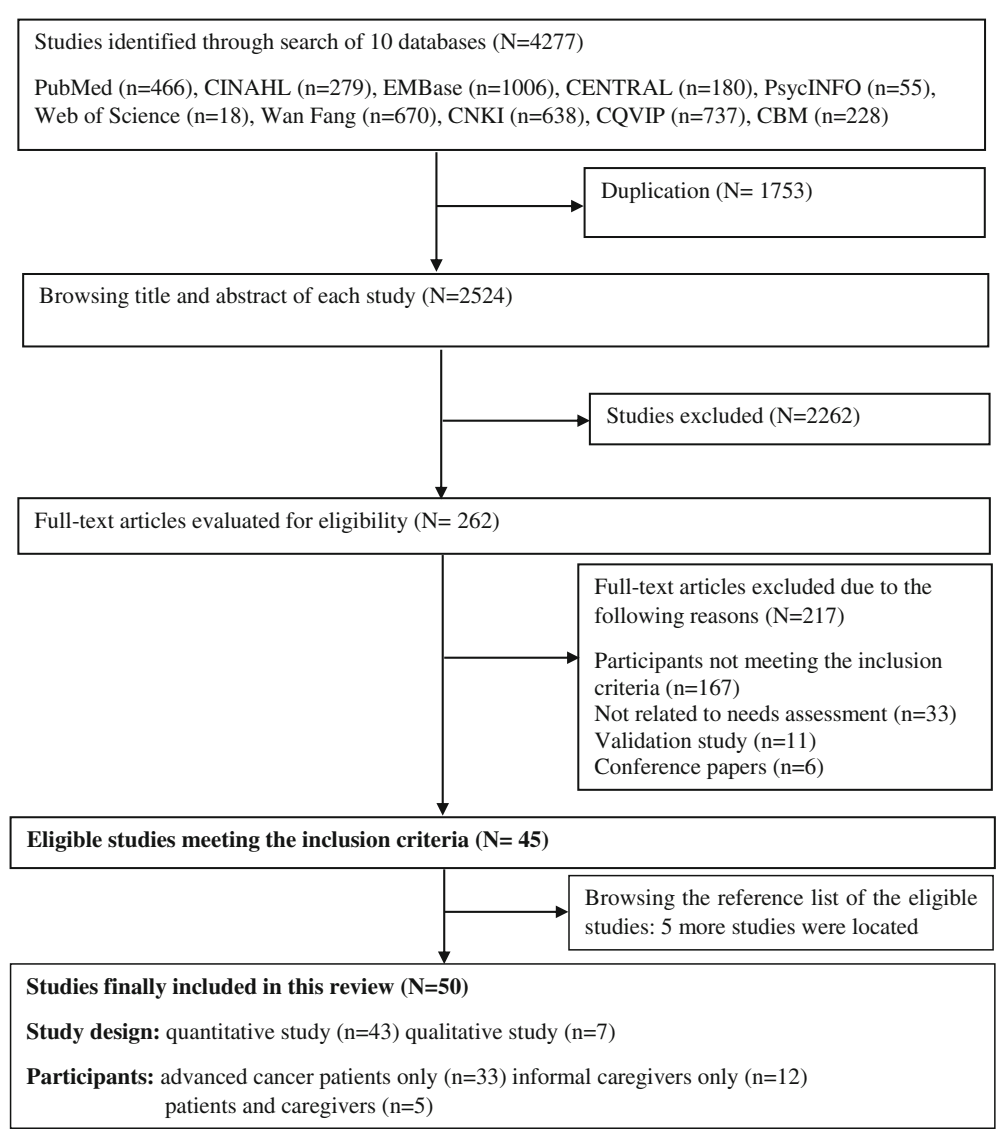

Fig. 1 Flow chart of study selection 
patients with advanced cancer only, with 31 out of 33 studies from the perspective of patients, one study from the perspective of informal caregivers, and one from the perspectives of both patients and informal caregivers. Twelve studies [9, 30, 32, 35, 39, 40, 49, 51, 52, 57, 62, 64] explored the unmet needs of informal caregivers, and five other [48, $56,59,63,67]$ studies investigated the unmet needs of patients with advanced cancer and their informal caregivers. With regards to sample sources, six studies $[32,40,45,46,49,61]$ reported no information regarding the recruitment setting, while in the remaining studies patients, and/or caregivers were mainly recruited from outpatient departments $(n=16)$, inpatient departments $(n$ $=11$ ), home/home-based care units $(n=10)$, and mixed settings $(n=7)$. In terms of cancer sites, 29 studies focused on patients with mixed cancer site and/or their caregivers, 11 studies focused on specific patients with cancer and/or caregivers (3 studies on prostate cancer [57, 69, 73], 5 studies on breast cancer $[41,48,58,60,75]$, and three on lung cancer $[35,42,71])$, while 10 other studies $[47,50$, $51,53,59,64,66,68]$ reported no information about cancer types. The diagnostic criteria of advanced cancer were presented in 13 studies (13/50), with five studies [6, 30, 31, $60,61]$ adopting the criteria of cancer with metastasis, and seven studies $[9,41,42,45,58,63,75]$ using the stage III/ IV criterion according to TNM staging system. With regards to geographic distribution, nine studies were conducted in the USA [38, 40, 46, 49, 52, 57, 59, 70, 74], seven were in mainland China (six of which were conducted in Shanghai) $[9,53,63-67]$, five in Australia $[6,54,55,60$, 68], five in the Netherlands [29-31, 34, 44], four in Canada $[47,50,56,73]$, three in Japan $[33,39,41]$, three in Taiwan $[35,42,62]$, two in the UK $[69,71]$, two in Denmark [45, 72], two in Hong Kong [58, 75], and one each in Italy [28], France [61], South Korea [32], Spain [37], Indonesia [36], Czech Republic [43], India [51], and Bangladesh [48]. Characteristics and main findings of all included studies are presented in Table 2.

\section{Quality of the included studies}

The methodological quality of the included studies was generally robust, with 17 and 18 studies satisfying all four criteria (34\%) and three of the four criteria (36\%), respectively. The prominent weaknesses of 43 quantitative studies were poor sampling strategy and low response rate. The response rates of 16 studies $[32,33,37,39,40,43,47,52-56,61,63,68,74]$ were lower than $60 \%$, and 14 studies [30-32, 38, 42, 43, $51-54,67,68,70,74]$ failed to report the sampling method, sampling procedure, or sample size justification. Among the seven other qualitative studies, three studies $(3 / 7,42.9 \%)[49,62,73]$ failed to interpret how findings were related to the study context, and two studies $(2 / 7,28.6 \%)[57,73]$ provided no explanation on how the research process was influenced by the researchers. The overall quality score of each study is presented in the first column of Table 2.

\section{Descriptions of unmet needs in patients with advanced cancer}

A total of 12 domains of unmet needs were identified from 34 quantitative and 4 qualitative studies. These domains included physical, ADL, psychological, health system and information, patient care and support, social, communication, financial, spiritual, autonomy, sexuality, and nutritional needs.

\section{Unmet patient needs based on quantitative studies}

Study sample sizes ranged from 40 to 977, with the average sample size being 165 and the response rate ranging from 36 to $100 \%$. Physical needs were reported in 24 studies, and the most prominent physical unmet need was fatigue [6, 31, 33, 34, 42, 43, 45, 47, 50, 54, 56, 63]. In terms of $\mathrm{ADL}, 11$ studies were included, and the most highlighted item was "not being able to do the things you used to do" [6, 33, 50, 58,60]. Twenty-eight studies reported psychological needs, and the most common item was "emotional support" $[6,28,29,31,33,36,41$, $45,46,50,70,72,73]$. In terms of health system and information, "being informed about benefits and side-effects of treatment" was the most common one $[31,41,42,44,54,61,63,66,69,75]$. With regards to patient care and support needs, two prominent unmet needs, namely, "reassurance by medical staff that the way you feel is normal" [33, 41] and "doctor acknowledges and shows sensitivity to your feelings and emotional needs" [33, 42], were identified. "Family and friends' support" was the most common social unmet need $[29,45,54,55,63,65,67]$. Communication and financial support needs were also reported $[28,29,31,36$, $43,46,54,56,63,66,55,70]$. "Meaning of death" [31, $36]$ was the most commonly mentioned spiritual need. "I can do less than before" [31, 34, 43] was the most prominent unmet autonomy need. Detailed unmet needs and their prevalence are presented in Table 3.

\section{Unmet patient needs extracted from qualitative studies}

According to four qualitative studies [48, 71-73], several unmet needs that were similar to those identified in quantitative studies were extracted and categorized. For instance, patients commonly expressed "pain, fatigue or side effects of treatment, such as urinary incontinence and loss of sexual function" (p. 191-192) (physical needs) [73], "feelings of fear, hopelessness and uncertainty about the future" $[48,71]$ or "feelings of sadness, anger, anxiety, frustration and desperation" [48, 71, 73] (psychological and spiritual needs), "insufficient information 
Table 2 Characteristics and Main Findings of the Included Studies

\begin{tabular}{|c|c|c|c|c|c|c|c|}
\hline $\begin{array}{l}\text { Author, Year } \\
\& Q S\end{array}$ & $\begin{array}{l}\text { Country/ } \\
\text { Region }\end{array}$ & Setting & Study Design & Participant & Diagnosis & $\begin{array}{l}\text { Response } \\
\text { Rate }\end{array}$ & $\begin{array}{l}\text { Data Collection Method/ } \\
\text { Instrument \& Findings }\end{array}$ \\
\hline \multicolumn{8}{|c|}{ Studies Regarding Advanced Cancer Patients $(n=33)$} \\
\hline \multirow[t]{2}{*}{$\begin{array}{l}\text { S1 [28]: } \\
\text { Morasso G, } \\
\text { et al., 1999, } \\
\text { QS:3 }\end{array}$} & \multirow[t]{2}{*}{ Italy } & \multirow[t]{2}{*}{ Inpatients } & \multirow[t]{2}{*}{$\begin{array}{l}\text { Semi-structured } \\
\text { interview survey }\end{array}$} & \multirow{2}{*}{$\begin{array}{l}\text { Sampling: } \\
\text { Random } \\
\text { sampling } \\
\text { Sample size: } \\
94 \\
\text { Age (yr): } 64.8 \\
\pm 11.1 \\
\text { Gender: } 38 / 89 \\
\text { (F) }\end{array}$} & \multirow[t]{2}{*}{$\begin{array}{l}\text { Terminal cancer } \\
\text { patients (mixed } \\
\text { cancer sites) }\end{array}$} & \multirow[t]{2}{*}{ 89/94 (94.7\%) } & $\begin{array}{l}\text { Interviews guide: } 5 \text { domains and } \\
41 \text { items: "physiological needs", } \\
\text { "safety needs", "loved and } \\
\text { belonging needs", "self-esteem } \\
\text { needs" and "self-fulfillment needs" } \\
\text { (p.404) }\end{array}$ \\
\hline & & & & & & & $\begin{array}{l}\text { Unmet needs (p.406): 1) symptoms } \\
\text { control (62.8\%), 2) occupational } \\
\text { functioning (62.1\%), 3) emotional } \\
\text { support }(51.7 \%), 4) \text { Nutrition } \\
(43.2 \%), 5) \text { sleep (37.1\%), 6) self- } \\
\text { fulfillment (32.5\%), 7) communica- } \\
\text { tion }(27.7 \%), 8) \text { information (25.0\%), } \\
\text { 9) personal care }(14.6 \%), 10) \text { finan- } \\
\text { cial support (14.1\%) and 11) emo- } \\
\text { tional closeness (13.8\%) }\end{array}$ \\
\hline $\begin{array}{l}\text { S2 [6]: } \\
\text { Waller, et al., } \\
2012, \\
\text { QS: } 4\end{array}$ & Australia & Outpatients & $\begin{array}{l}\text { Multiple time } \\
\text { points pre-post } \\
\text { intervention } \\
\text { study }^{\text {a }}\end{array}$ & $\begin{array}{l}\text { Sampling: } \\
\text { unclear } \\
\text { (219/613) } \\
\text { Sample size: } \\
219 \\
\text { Age (yr): } 66.1\end{array}$ & $\begin{array}{l}\text { Advanced cancer } \\
\text { patients (extensive } \\
\text { local, regional or } \\
\text { metastatic) (mixed } \\
\text { cancer sites) }\end{array}$ & $\begin{array}{l}195 / 219 \\
(89.0 \%)\end{array}$ & $\begin{array}{l}\text { Supportive Care Needs Survey } \\
\text { (SCNS-SF34): } 5 \text { domains and } 34 \\
\text { items } \\
\text { Needs Assessment for Advanced } \\
\text { Cancer Patients (NA-ACP): only } \\
\text { used } 6 \text { items on spiritual needs }\end{array}$ \\
\hline
\end{tabular}

$\pm 10.7$

Gender: 91/

$195(\mathrm{~F})$

$\begin{array}{lllll}\text { S3 [29]: } & \text { Netherla- } & \text { Inpatients } & \begin{array}{l}\text { Structured } \\ \text { interview survey }\end{array} & \text { Sampling: } \\ \text { Teunissen, } & \text { nds } & & & \text { Snclear } \\ \text { SC, et al., } & & & \text { Sample size: } \\ 2006 & & & 181 \\ \text { QS: } 3 & & \text { Age (median, } \\ & & \text { yr): } 18-79 \\ & & \text { Gender: } 101 / \\ & & 181(\mathrm{~F})\end{array}$

S5 [31]: Osse Netherla- HomeBHP, et al., nds based 2005,

QS: 3

Questionnaire
survey

Sampling: unclear?

Sample size:

112

Age (yr): $58 \pm$

$12.3(30-87)$
Advanced cancer $\quad$ 181/181 patients (mixed (100\%) cancer sites)

Distant metastatic cancer (mixed cancer sites)
$94 / 112$

(84.0\%)

Moderate-to-high unmet needs: 1) "not being able to do the things you used to do" (33.0\%), 2) "concerns about the worries of those close to you" $(27.9 \%), 3)$ "lack of energy, tiredness" (26.2\%), 4) "work around the home" (23.0\%), 5) "uncertainty about the future"(21.4\%), 6) "pain" (20.9\%), 7) "worry that results of treatment are beyond your control" (19.4\%), 8) "fears about the cancer spreading" $(18.8 \%), 9)$ "felling unwell a lot of the time"(17.3\%), and 10) "anxiety" (15.3\%)

Structured interview with a standard list: 4 domains: emotional needs, social needs, spiritual needs, and functional needs. (p.153) Each item including 2 parts: 1 ) if the issue is a "problem"; 2) actual wishes to receive professional support were labelled as palliative care needs. (p. 153)

Unmet needs:

1) functional support $(62.4 \%), 2)$ support in coping $(57.5 \%), 3)$ emotional support $(53.1 \%), 4)$ support of informal caregivers (34.3\%), 5) spiritual support (7.7\%), 6) co-ordination of care $(9.9 \%), 7$ ) relational support (9.9\%), and 8 ) support in communication (7.7\%).

Problems and Needs in Palliative Care questionnaire (PNPC): 10 domains and 90 items

Top 10 unmet needs: 1) "difficulty coping with the unpredictability of the future" (25\%), 2) "fear of metastases" (25\%), 3) "fear of 
Table 2 Characteristics and Main Findings of the Included Studies (Continued)

Author, Year Country/ Setting Study Design Participant Diagnosis

\& QS

Region

Gender: 66/94

(F)

\section{S7 [33]:}

Hasegawa,

et al., 2016

QS: 3

\section{Japan \\ Inpatients \\ Questionnaire}

survey
S8 [34]:

Uitdehaag

MJ et al.,

2015

QS: 4

\section{Netherla- Outpatients Questionnaire nds survey}

Sampling:
random
sampling
Sample size:
45
Age (yr): 66.6
\pm 9.8
Gender: $21 / 45$

Gende

(F)
Advanced cancer $\quad$ NR patients (mixed cancer sites)

Response

Rate

(n)

(n)


Table 2 Characteristics and Main Findings of the Included Studies (Continued)

\begin{tabular}{|c|c|c|c|c|c|c|c|}
\hline $\begin{array}{l}\text { Author, Year } \\
\& \text { QS }\end{array}$ & $\begin{array}{l}\text { Country/ } \\
\text { Region }\end{array}$ & Setting & Study Design & Participant & Diagnosis & $\begin{array}{l}\text { Response } \\
\text { Rate }\end{array}$ & $\begin{array}{l}\text { Data Collection Method/ } \\
\text { Instrument \& Findings }\end{array}$ \\
\hline \multirow[t]{2}{*}{ QS: 2} & & & & \multirow{2}{*}{$\begin{array}{l}\text { Sample size: } \\
180 \\
\text { Age (yr): } \\
\text { Indonesian: } \\
49.3 \pm 10.7 \\
\text { Netherlands: } \\
58 \pm 12.3 \\
\text { Gender: } \\
\text { Indonesian: } \\
\text { 133/180 (F) } \\
\text { Netherlands: } \\
66 / 94 \text { (F) }\end{array}$} & & & $\begin{array}{l}\text { adjusted within Indonesian context } \\
\text { and deleted } 9 \text { items, and } 24 \text { items } \\
\text { were maintained }\end{array}$ \\
\hline & & & & & & & $\begin{array}{l}\text { Unmet needs: } \\
\text { Physical: sweating (76.2\%), sexuality } \\
\text { ( } 75 \%) \text {, short of breathless ( } 67.3 \%), \\
\text { pain (66.4\%) } \\
\text { Autonomy: "difficulties in finding } \\
\text { someone to talk to" }(82.8 \%) \text {; } \\
\text { Psychological: "difficulties showing } \\
\text { emotions" (84.4\%) } \\
\text { Spiritual: "difficulties about the } \\
\text { meaning of death" (85.4\%) } \\
\text { Financial: "extra expenses because } \\
\text { of the disease" (72\%) }\end{array}$ \\
\hline \multirow{2}{*}{$\begin{array}{l}\text { S11 [37]: } \\
\text { Vilalta, A, et } \\
\text { al., } \\
2014 \\
\text { QS: } 3\end{array}$} & \multirow[t]{2}{*}{ Spain } & \multirow[t]{2}{*}{ Outpatients } & \multirow[t]{2}{*}{$\begin{array}{l}\text { Questionnaire } \\
\text { survey }\end{array}$} & \multirow{2}{*}{$\begin{array}{l}\text { Sampling: } \\
\text { unclear } \\
\text { Sample size: } \\
50 \\
\text { Age (yr): } \\
\text { Mean } 60.9 \\
\text { (33-81) } \\
\text { Gender: 19/50 } \\
\text { (F) }\end{array}$} & \multirow{2}{*}{$\begin{array}{l}\text { Advanced cancer } \\
\text { (mixed cancer sites) }\end{array}$} & \multirow[t]{2}{*}{ NR } & $\begin{array}{l}\text { Self-designed questionnaire for } \\
\text { spiritual needs: } 11 \text { domains and } 28 \\
\text { items }\end{array}$ \\
\hline & & & & & & & $\begin{array}{l}\text { Top } 10 \text { spiritual needs (p. 594): } \\
\text { 1) "to be recognized as a person } \\
\text { until the end of life" ( } 8.6 \pm 1.3) ; 2) \\
\text { "the need for truth" }(8.3 \pm 2.7) ; 3) \\
\text { "to reinterpret life" (6.2 } 2 \pm 1.9) ; 4) \text { "to } \\
\text { look for a meaning to existence" } \\
(5.7 \pm 2.5) ; 5) \text { "the need for hope" } \\
(5.7 \pm 3.5) ; 6) \text { "to see life beyond } \\
\text { the individual" (5.2 } \pm 2.5) ; 7) \text { "the } \\
\text { need for religious expression" ( } 4.9 \\
\pm 2.5) ; 8) \text { "the needs for continuity } \\
\text { and an afterlife" (4.0 } \pm 2.0) ; 9) \text { "the } \\
\text { need for freedom and to be free" } \\
\text { (3.8 } \pm 3.4) ; 10) \text { "to be free blame } \\
\text { and to forgive others" (1.5 } \pm 2.0) \text {. }\end{array}$ \\
\hline \multirow[t]{2}{*}{$\begin{array}{l}\text { S12 [38]: } \\
\text { Schenker Y. } \\
\text { et al., } \\
2014 \\
\text { QS: } 3\end{array}$} & \multirow[t]{2}{*}{ USA } & \multirow[t]{2}{*}{ Outpatients } & \multirow[t]{2}{*}{$\begin{array}{l}\text { Questionnaire } \\
\text { survey }\end{array}$} & \multirow{2}{*}{$\begin{array}{l}\text { Sampling: } \\
\text { unclear } \\
\text { Sample size: } \\
169 \\
\text { Age (yr): } 62.3 \\
\pm 11.6 \\
\text { Gender: 107/ } \\
169 \text { (F) }\end{array}$} & \multirow[t]{2}{*}{$\begin{array}{l}\text { Advanced cancer } \\
\text { (mixed cancer sites) }\end{array}$} & \multirow[t]{2}{*}{$\begin{array}{l}169 / 272 \\
(62.1 \%)\end{array}$} & $\begin{array}{l}\text { Adapted Needs Assessment of } \\
\text { Advanced Cancer Patients (NA- } \\
\text { ACP): } 32 \text { items and } 6 \text { domains, } \\
\text { without reporting psychological } \\
\text { properties }\end{array}$ \\
\hline & & & & & & & $\begin{array}{l}\text { Unmet needs: 1) symptom (62\%); } \\
\text { 2) psychological }(62 \%) ; 3) \text { medical } \\
\text { communication/information (39\%); } \\
\text { 4) daily living }(27 \%) ; 5) \text { spiritual } \\
(23 \%) ; 6) \text { social }(20 \%)\end{array}$ \\
\hline \multirow[t]{2}{*}{$\begin{array}{l}\text { S16 [41]: } \\
\text { Uchida M, et } \\
\text { al., } 2011 \\
\text { QS: } 4\end{array}$} & \multirow[t]{2}{*}{ Japan } & \multirow[t]{2}{*}{ Outpatients } & \multirow[t]{2}{*}{$\begin{array}{l}\text { Questionnaire } \\
\text { survey }\end{array}$} & \multirow{2}{*}{$\begin{array}{l}\text { Sampling: } \\
\text { random } \\
\text { sampling } \\
\text { Sample size: } \\
85 \\
\text { Age (yr): } 58.6 \\
\pm 11.9 \\
\text { Gender: } 85 / 87 \\
\text { (F) }\end{array}$} & \multirow[t]{2}{*}{$\begin{array}{l}\text { Advanced breast } \\
\text { cancer patients } \\
\text { (stage IV) }\end{array}$} & \multirow[t]{2}{*}{$\begin{array}{l}85 / 87 \\
(97.7 \%)\end{array}$} & $\begin{array}{l}\text { Supportive Care Needs Survey } \\
\text { (SCNS-SF34): } 5 \text { domains and } 34 \\
\text { items } \\
\text { Hospital Anxiety and Depression } \\
\text { Scale (HADS) } \\
\text { EOERC-QLQ-C30 }\end{array}$ \\
\hline & & & & & & & $\begin{array}{l}\text { Top } 10 \text { moderate-to-high unmet } \\
\text { needs: } \\
\text { 1) "Fears about the cancer } \\
\text { spreading" (78.8\%); } 2 \text { ) "Worry that } \\
\text { the results of treatment are } \\
\text { beyond your control" (71.8\%); } 3) \\
\text { "Concerns about the worries of } \\
\text { those close to you" (68.2\%); } 4) \\
\text { "Having one member of hospital } \\
\text { staff with whom you can talk to } \\
\text { about all aspects of your condition, } \\
\text { treatment and follow-up" (67.1\%); } \\
\text { 5) "Being informed about things } \\
\text { you can do to help yourself to get }\end{array}$ \\
\hline
\end{tabular}


Table 2 Characteristics and Main Findings of the Included Studies (Continued)

Author, Year Country/ Setting Study Design Participant Diagnosis

Region

Setting

Response
Rate

Data Collection Method/ Instrument \& Findings

well" (65.9\%); 6) "Anxiety" (65.9\%); 7) "Feeling down or depressed" (62.4\%); 8) "Uncertainty about the future" (62.4\%); 9) "Feeling about death and dying" (62.4\%); 10) "Having access to professional counseling if you, family or friends need it" (57.6\%);

\begin{tabular}{|c|c|c|c|c|}
\hline $\begin{array}{l}\text { S17 [42]: } \\
\text { Liao YC, et } \\
\text { al., } \\
2011 \\
\text { QS: } 3\end{array}$ & Taiwan & Mixed & $\begin{array}{l}\text { Questionnaire } \\
\text { survey }\end{array}$ & $\begin{array}{l}\text { Sampling: } \\
\text { unclear } \\
\text { Sample size: } \\
152 \\
\text { Age (yr): } 60.2 \\
\pm 11.0 \\
\text { Gender: } 73 / \\
152 \text { (F) }\end{array}$ \\
\hline
\end{tabular}

Advanced lung $\quad 152 / 188$

cancer patients $\quad$ (80.9\%) (95.4\% stage III-IV or extensive metastasis)
S18 [43]:

BUŽGOVÁ,

et al., 2014

QS: 2

\section{Czech \\ Republic}

Inpatients

Questionnaire survey
Sampling:

Sample size: 93

Age (yr): 61.6

$\pm 16.8$

Gender: 41/93

(F)
Advanced cancer $\quad$ NR

(mixed cancer sites)
Cancer Needs Questionnaire (CNQ)-Chinese version: 5 domains and32 items

Hospital Anxiety and Depression Scale (HADS)

Symptom Severity Scale (SSS)

Items of highest unmet needs by each domain:

1) "things helping self get well" (65.8\%), 2) "cancer remission" (63.8\%), 3) "benefit and side-effects of treatment" (63.8\%), 4) "test results as soon as possible" (62.5\%); 5) "dealing with fears about disease spreading and return" (40.2\%), 6) "doctor acknowledges and shows sensitivity to your feelings and emotional needs" (39.5\%), 7) "dealing with lack of energy and tiredness" (28.3\%)

Patient Needs Assessment in Palliative Care (PNAP): 5 domains and 42 items

Hospital Anxiety and Depression Scale (HADS)

EOERC-QLQ-C30

Items of highest unmet needs by each domain:

1)Spiritual: "attending religious services or other ceremonies" (44\%); 2) Autonomy: "continue my usual activities" (38\%); 3) Social: "being financially secure" (27\%); 4) psychological: "fear of dependence on help from others" (30\%); 5) physical: "fatigue" (30\%);

Problems and Needs in Palliative Care questionnaire (PNPC): used the 12 items on information needs Hospital Anxiety and Depression Scale (HADS)

Utrecht Coping List to measure disease-specific coping

Unmet information: 1) complementary care $(93 \%) ; 2$ ) alternative medicine (86\%); 3 ) euthanasia: (83\%); 4) care settings (78\%); 5) Sexuality and cancer $(72 \%) ; 6)$ psychological care $(71 \%)$; 7) cause of cancer (65\%); 8) food and diet (44\%); 9) helpful devices (33\%): 10) organizations that offer help (32\%); 11) expected physical (20\%); 12) treatment options and side effects (4\%) 
Table 2 Characteristics and Main Findings of the Included Studies (Continued)

\begin{tabular}{|c|c|c|c|c|c|c|}
\hline $\begin{array}{l}\text { Author, Year } \\
\& Q S\end{array}$ & $\begin{array}{l}\text { Country/ } \\
\text { Region }\end{array}$ & Setting & Study Design & Participant & Diagnosis & $\begin{array}{l}\text { Response } \\
\text { Rate }\end{array}$ \\
\hline $\begin{array}{l}\text { S20 [45]: } \\
\text { Johnsen AT, } \\
\text { et al., } \\
2013 \\
\text { QS: } 4\end{array}$ & Denmark & NR & $\begin{array}{l}\text { Questionnaire } \\
\text { survey }\end{array}$ & $\begin{array}{l}\text { Sampling: } \\
\text { random } \\
\text { sampling } \\
\text { Sample size: } \\
977 \\
\text { Age (yr): } \\
\text { mean } 64 \\
\text { Gender: 547/ } \\
977 \text { (F) }\end{array}$ & $\begin{array}{l}\text { Advanced cancer } \\
\text { with mixed sites } \\
\text { (95\% at stage III/ IV) }\end{array}$ & $\begin{array}{l}977 / 1630 \\
(60 \%)\end{array}$ \\
\hline
\end{tabular}

Data Collection Method/

Instrument \& Findings

\begin{tabular}{|c|c|c|c|c|c|}
\hline $\begin{array}{l}\text { S21 [46]: } \\
\text { Houts P, et } \\
\text { al., } 1988 \\
\text { QS: } 4\end{array}$ & USA & NR & $\begin{array}{l}\text { Semi-structured } \\
\text { interview survey } \\
\text { (retrospective) }\end{array}$ & $\begin{array}{l}\text { Sampling: } \\
\text { stratified } \\
\text { random } \\
\text { sampling } \\
\text { Sample size: } \\
433 \\
\text { Age (yr): } \geq 20 y \\
\text { Gender: } \\
\text { unclear }\end{array}$ & $\begin{array}{l}\text { Caregivers of } \\
\text { terminal cancer } \\
\text { (mixed cancer sites) }\end{array}$ \\
\hline
\end{tabular}

\begin{tabular}{|c|c|c|c|c|c|}
\hline $\begin{array}{l}\text { S22 [47]: } \\
\text { Khan L, et } \\
\text { al., } \\
2012 \\
\text { QS: } 3\end{array}$ & Canada & Outpatients & $\begin{array}{l}\text { Questionnaire } \\
\text { survey }\end{array}$ & $\begin{array}{l}\text { Sampling: } \\
\text { unclear } \\
\text { Sample size: } \\
40 \text { (patients= } \\
20 \text {, caregivers } \\
=20 \text { ) } \\
\text { Age (yr): } \\
\text { Patients: } \\
\text { unclear } \\
\text { Caregivers: } \\
\text { unclear } \\
\text { Gender: }\end{array}$ & $\begin{array}{l}\text { Advanced cancer } \\
\text { patients and their } \\
\text { caregivers (cancer } \\
\text { site unclear) }\end{array}$ \\
\hline
\end{tabular}

\begin{tabular}{|c|c|c|c|c|c|c|c|}
\hline $\begin{array}{l}\text { S25 [50]: } \\
\text { Fitch MI, } \\
2012 \\
\text { QS: } 4\end{array}$ & Canada & Outpatients & $\begin{array}{l}\text { Questionnaire } \\
\text { survey }\end{array}$ & $\begin{array}{l}\text { Sampling: } \\
\text { convenience } \\
\text { sampling } \\
\text { Sample size: } \\
69\end{array}$ & $\begin{array}{l}\text { Advanced cancer } \\
\text { patients (cancer } \\
\text { sites unclear) }\end{array}$ & $\begin{array}{l}69 / 106 \\
(65.1 \%)\end{array}$ & $\begin{array}{l}\text { Adapted Supportive Care Needs } \\
\text { Survey (SCNS): } 7 \text { domains and } 61 \\
\text { items: information, physical } \\
\text { symptoms, psychological, } \\
\text { emotional, spiritual, social, and } \\
\text { practical, Cronbach's } a=0.35-0.81\end{array}$ \\
\hline
\end{tabular}


Table 2 Characteristics and Main Findings of the Included Studies (Continued)

\begin{tabular}{|c|c|c|c|c|c|c|c|}
\hline $\begin{array}{l}\text { Author, Year } \\
\& \text { QS }\end{array}$ & $\begin{array}{l}\text { Country/ } \\
\text { Region }\end{array}$ & Setting & Study Design & Participant & Diagnosis & $\begin{array}{l}\text { Response } \\
\text { Rate }\end{array}$ & $\begin{array}{l}\text { Data Collection Method/ } \\
\text { Instrument \& Findings }\end{array}$ \\
\hline & & & & $\begin{array}{l}\text { Age (yr): } \\
\text { mean 65y (35- } \\
84 y) \\
\text { Gender: } 34 / 69 \\
\text { (F) }\end{array}$ & & & $\begin{array}{l}\text { Unmet needs in terms of issues } \\
\text { reported by } 50 \% \text { patients: } \\
\text { 1) "pain" (63.5\%); 2) "fear of pain" } \\
(62.9 \%) ; 3) \text { "lack of energy" ( } 52.8 \%) ; \\
\text { 4) "fear about physical disability or } \\
\text { deterioration" (50\%); 5) "fear about } \\
\text { cancer spreading" (51.4\%;); } 6 \text { ) "not } \\
\text { being able to do things you used } \\
\text { to" (46.9\%); 7) "decreased appetite" } \\
\text { (47.4\%); 8) "feeling unwell" (44.7\%); } \\
\text { 8) "feeling down or depressed" } \\
\text { (30\%), 9) "not being able to work } \\
\text { around at home" ( } 44.2 \%) ; 10) \\
\text { "concerns about the worries of } \\
\text { those close to you"(29.4\%) }\end{array}$ \\
\hline
\end{tabular}

\begin{tabular}{|c|c|c|c|c|}
\hline $\begin{array}{l}\text { S28 [53]: } \\
\text { Deng D, et } \\
\text { al. } 2015 \\
\text { QS: } 2\end{array}$ & China & $\begin{array}{l}\text { Home- } \\
\text { based }\end{array}$ & Interview survey & $\begin{array}{l}\text { Sampling: } \\
\text { unclear } \\
\text { Sample size: } \\
107 \\
\text { Age (yr): } \\
\text { mean 57y (18- } \\
87 y \text { ) } \\
\text { Gender: 58/ } \\
107 \text { (F) }\end{array}$ \\
\hline
\end{tabular}

\section{S29 [54]:}

Rachakonda

$\mathrm{K}$, et al.,

2015

QS: 1

Australia Inpatients $\begin{aligned} & \text { Questionnaire } \\ & \text { survey }\end{aligned}$

unclear

Eligible

sample:

unclear

Sample

size: 75

Age (yr): $68 \pm$

12

Gender: 32/75

(F)

\author{
Advanced cancer \\ patients (cancer \\ sites unclear)

NR

Items of highest unmet needs by each domain:

1) symptom "dealing with lack of energy or tiredness" (30.7\%); 2)

psychological "coping with frustration at not being able to do the things you used to do" (24.3\%); 3) daily livings "getting assistance with preparing meals" (12\%); 4) social "receiving emotional support from friends and family" (12.2\%); 5) medical information and communication (9.3-14.9\%) "getting information about nonconventional treatments" (14.9\%); 6) financial "paying the nonmedical costs of your illness"; $(17.3 \%)$; 7) spiritual "being able to choose the place where you want to die" (11\%).

\begin{tabular}{|c|c|c|c|c|c|}
\hline $\begin{array}{l}\text { S30 [55]: } \\
\text { Rainbird K, } \\
\text { et al. } 2009 \\
\text { QS: } 3\end{array}$ & Australia & $\begin{array}{l}\text { Home- } \\
\text { based }\end{array}$ & $\begin{array}{l}\text { Questionnaire } \\
\text { survey }\end{array}$ & $\begin{array}{l}\text { Sampling: } \\
\text { unclear } \\
\text { Sample size: } \\
246 \\
\text { Age (yr): } 61 \pm \\
11.9 \\
\text { Gender: } 131 / \\
246 \text { (F) }\end{array}$ & $\begin{array}{l}\text { Advanced cancer } \\
\text { patients (mixed } \\
\text { cancer sites) }\end{array}$ \\
\hline
\end{tabular}

\section{Needs Assessment of Advanced Cancer Patients (NA-ACP): 7 domains and 132 items}

Items of highest unmet needs by each domain:

1) symptom (15-22\%)' "dealing with loss of appetite" (22\%); 2) psychological (39-40\%), "coping with fears about the caner spreading" (40\%) and "coping with frustration at not being able to do the things you used to do" (40\%); 3) daily livings (10-30\%), "dealing with doing work around the house" (30\%); 4) social (10-13\%), 
Table 2 Characteristics and Main Findings of the Included Studies (Continued)

Author, Year Country/ Setting Study Design Participant Diagnosis

Region

S33 [58]:

Au A, et al.,

Hong Kong Outpatients

2013,

QS: 4

S35 [60]:

Australia

Aranda S, et

al., 2005

QS: 4
Sampling: consecutive sampling

Sample size:

198

Age (yr): 53.4

$\pm 9.74$

Gender: 198/

198 (F)
Advanced breast cancer patients (stage III/IV)
$198 / 220$

(90\%)
Response

Rate
Data Collection Method/

Instrument \& Findings

"being able to express feeling with friends and/or family" (13\%); 5) medical information and communication (31-35\%), "getting information about factors, which could influence the course of the cancer" (35\%); 6) financial (11$12 \%)$, "dealing with concerns about your financial situation" (12\%); 7 ) spiritual (11-15\%), "being able to choose the place where you want to die" $(15 \%)$

Chinese version of Supportive Care Needs Survey (SCNS-SF33-C): 4 domains and 33 items: physical and daily living, psychological, sexuality, health system, information and patient support (HSIPS)

Hospital Anxiety and Depression Scale (HADS)

Memorial Symptom Assessment Scale Short-Form (MSAS-SF)

Chinese Patient Satisfaction Questionnaire

Top 10 moderate-to-high unmet needs:

1) "Having one member of hospital staff with whom you can talk to about your concerns" (63.7\%); 2) "informed about cancer is under control or diminishing" (61.6\%); 3 ) "Informed about things you can do to get well" (58.6\%); 4)

"Informed about your test results" (51\%); 5) "Given written information" (46.9\%); 6) "given information about aspects of managing illness and side-effects at home" (39.9\%); 7 ) "adequately information about the benefits and side-effects of treatments" (39.3\%); 8) "given explanations of those tests for which you would like explanations" (36.9\%); 9) "being treated like a person" (35.4\%); 10) "more choice about cancer specialists" (31.8\%)

Sampling: consecutive sampling Sample size: 105 Age (yr): (3485 , median 57) Gender: 105/ 105(F)
Metastatic breast cancer

$105 / 172$

(61\%)
Supportive Care Needs
Questionnaire (SCNQ): 5 domains and 59 items

Moderate to high unmet needs: 1)Psychological needs (24-41\%): "concerns about the worries of those close to you" (41\%),

"uncertainty about the future" (38\%), etc.

2)Information needs (26-41\%): "informed about things you can do to help yourself get well" (41\%), "one member of hospital staff with whom you can talk" (32\%), etc. 3)Physical and daily living needs (25-28\%): "pain" (28\%), "not being 
Table 2 Characteristics and Main Findings of the Included Studies (Continued)

Author, Year Country/ Setting Study Design Participant Diagnosis

\& QS

Region

Seting

Study Design a Paticipant Diagnosis

Response
Rate

Data Collection Method/

Instrument \& Findings

S36 [61]:
Lelorain S,
et al., 2015
QS: 2

France NR

NR Questionnaire

survey

S40 [65]: Gu Shanghai, Inpatients

WJ, et al., China

2015

QS: 3

survey

\section{survey}

S41 [66]

Huang $J$, et

Shanghai,

Home-

al., 2008

QS: 3

China based

Australia Mixed

Waller $A$, et

al., 2012

QS: 2

\section{Sampling: consecutive sampling \\ Sample size: 201 \\ Age (yr): \\ mean 62 \\ Gender: 146/ \\ $201(F)$}

\section{Metastatic cancer NR (mixed cancer sites)}

.


Table 2 Characteristics and Main Findings of the Included Studies (Continued)

\begin{tabular}{|c|c|c|c|c|c|c|c|}
\hline $\begin{array}{l}\text { Author, Year } \\
\& \text { QS }\end{array}$ & $\begin{array}{l}\text { Country/ } \\
\text { Region }\end{array}$ & Setting & Study Design & Participant & Diagnosis & $\begin{array}{l}\text { Response } \\
\text { Rate }\end{array}$ & $\begin{array}{l}\text { Data Collection Method/ } \\
\text { Instrument \& Findings }\end{array}$ \\
\hline & & & & $\begin{array}{l}\text { Age (yr): } 66.1 \\
\pm 10.7 \\
\text { Gender: } 90 / \\
198 \text { (F) }\end{array}$ & & & $\begin{array}{l}\text { 2) daily living: } 29 \% \\
\text { 3) psychological: } 19 \%\end{array}$ \\
\hline \multirow{2}{*}{$\begin{array}{l}\text { S44 [69]: } \\
\text { Templeton, } \\
\text { H, et al., } \\
2003 \\
\text { QS: } 4\end{array}$} & \multirow[t]{2}{*}{ UK } & \multirow[t]{2}{*}{$\begin{array}{l}\text { Home- } \\
\text { based }\end{array}$} & \multirow[t]{2}{*}{$\begin{array}{l}\text { Structured } \\
\text { interview survey }\end{array}$} & \multirow{2}{*}{$\begin{array}{l}\text { Sampling: } \\
\text { unclear } \\
\text { Sample size: } \\
90 \\
\text { Age (yr): } 71- \\
80(48.9 \%) \\
\text { Gender: } 90 \\
\text { (M) }\end{array}$} & \multirow[t]{2}{*}{$\begin{array}{l}\text { Advance prostate } \\
\text { cancer }\end{array}$} & \multirow[t]{2}{*}{$79 \%$} & $\begin{array}{l}\text { Adapted Toronto Information } \\
\text { Needs Questionnaire (TINQ-BC): } 5 \\
\text { domains and } 29 \text { items }\end{array}$ \\
\hline & & & & & & & $\begin{array}{l}\text { Unmet needs: } \\
82.2 \% \text { of the patients need more } \\
\text { information: } \\
\text { 1) "side effects of treatment" } \\
\text { ( } 66.7 \%) \text {; } \\
\text { 2) "how to ease side effects of } \\
\text { treatment" (64.4\%) }\end{array}$ \\
\hline \multirow{2}{*}{$\begin{array}{l}\text { S45 [70]: } \\
\text { Hwang, S, et } \\
\text { al., } 2004 \\
\text { QS:3 }\end{array}$} & \multirow[t]{2}{*}{ USA } & \multirow[t]{2}{*}{ Mixed } & \multirow[t]{2}{*}{$\begin{array}{l}\text { Questionnaire } \\
\text { survey }\end{array}$} & \multirow{2}{*}{$\begin{array}{l}\text { Sampling: } \\
\text { consecutive } \\
\text { sampling } \\
\text { Sample size: } \\
296 \\
\text { Age (yr): } \\
\text { median } 68 \\
\text { (29-96) } \\
\text { Gender: } 296 \\
\text { (M) }\end{array}$} & \multirow[t]{2}{*}{$\begin{array}{l}\text { Advance cancer } \\
\text { (mixed cancer sites) }\end{array}$} & \multirow[t]{2}{*}{$\begin{array}{l}296 / 312 \\
(94.9 \%)\end{array}$} & $\begin{array}{l}\text { 14-item unmet needs } \\
\text { questionnaire: } 5 \text { domains and } 14 \\
\text { items }\end{array}$ \\
\hline & & & & & & & $\begin{array}{l}\text { Unmet needs: } \\
\text { 1) physical: } 46.1-80 \% \text {; } \\
\text { 2) emotional/social: } 10.1-32.5 \% \\
\text { 3) economic: } 6.6-17.3 \% \\
\text { 4) medical: } 12.5-13.6 \% \\
\text { 5) community: } 0-14.3 \%\end{array}$ \\
\hline \multirow{2}{*}{$\begin{array}{l}\text { S46 [71]: } \\
\text { Murray, SA, } \\
\text { et al., 2004 } \\
\text { QS: } 4\end{array}$} & \multirow[t]{2}{*}{ UK } & \multirow[t]{2}{*}{ Outpatients } & \multirow[t]{2}{*}{$\begin{array}{l}\text { Semi-structured } \\
\text { interview }\end{array}$} & \multirow{2}{*}{$\begin{array}{l}\text { Sampling: } \\
\text { purposive } \\
\text { sampling } \\
\text { Sample size: } \\
20 \\
\text { Age (yr): } \\
\text { median } 65 \\
\text { Gender: } \\
\text { unclear }\end{array}$} & \multirow[t]{2}{*}{$\begin{array}{l}\text { Advance lung } \\
\text { cancer }\end{array}$} & \multirow[t]{2}{*}{ NA } & $\begin{array}{l}\text { Semi-structured interview, } 40 \text { mins- } \\
2 \mathrm{~h} \text {, tape recorded }\end{array}$ \\
\hline & & & & & & & $\begin{array}{l}\text { Unmet needs: } \\
\text { 1) "fear, distress and uncertainty" } \\
\text { (p. 41) } \\
\text { 2) review "what they had achieved, } \\
\text { what still needed to be done } \\
\text { before death" (p. 42), and establish } \\
\text { themselves as they 'really' are (p. } \\
\text { 41) } \\
\text { 3) "feeling of loss of control" (p. 42) } \\
\text { 4) "hard to find hope," and } \\
\text { "questioned their faith wonder } \\
\text { why God had not heeded their } \\
\text { prayers" (p.42) }\end{array}$ \\
\hline \multirow{2}{*}{$\begin{array}{l}\text { S47 [72]: } \\
\text { Soelver L, et } \\
\text { al., } 2014 \\
\text { QS: } 4\end{array}$} & \multirow[t]{2}{*}{ Denmark } & \multirow[t]{2}{*}{ Inpatients } & \multirow[t]{2}{*}{$\begin{array}{l}\text { Semi-structured } \\
\text { interview }\end{array}$} & \multirow{2}{*}{$\begin{array}{l}\text { Sampling: } \\
\text { open and } \\
\text { strategic } \\
\text { sampling } \\
\text { Sample size: } \\
11 \\
\text { Age (yr): } \\
\text { median } 71.3 \\
\text { (54-86) } \\
\text { Gender: } 7 / 11 \\
\text { (F) }\end{array}$} & \multirow[t]{2}{*}{$\begin{array}{l}\text { Advance cancer } \\
\text { (mixed cancer sites) }\end{array}$} & \multirow[t]{2}{*}{ NA } & $\begin{array}{l}\text { Semi-structured interview, 30mins- } \\
1 \mathrm{~h}\end{array}$ \\
\hline & & & & & & & $\begin{array}{l}\text { Unmet needs (pp. 177-180): } \\
\text { 1) professionals failed to provide } \\
\text { patients timely information; 2) } \\
\text { patients experienced that } \\
\text { "professionals failed to give much } \\
\text { help in terms of physical and } \\
\text { emotional burden"; } 3 \text { ) Not being } \\
\text { regarded as a person: "lack of } \\
\text { dialogue with professionals make } \\
\text { patients feel neglected and } \\
\text { uncertain in the sense of } \\
\text { belonging"; 4) autonomy: "patients } \\
\text { wanted to be proactive in problem } \\
\text { solving, but did not know how to } \\
\text { do"; 5) lack of help for their } \\
\text { physical and emotional problem }\end{array}$ \\
\hline \multirow{2}{*}{$\begin{array}{l}\text { S48 [73]: } \\
\text { Cater N, et } \\
\text { al., } 2011 \\
\text { QS: } 2\end{array}$} & \multirow[t]{2}{*}{ Canada } & \multirow[t]{2}{*}{ Outpatients } & \multirow{2}{*}{$\begin{array}{l}\text { Semi-structured } \\
\text { focus group and } \\
\text { in-depth } \\
\text { interview }\end{array}$} & \multirow{2}{*}{$\begin{array}{l}\text { Sampling: } \\
\text { unclear } \\
\text { Sample size: } \\
29\end{array}$} & \multirow[t]{2}{*}{$\begin{array}{l}\text { Advance prostate } \\
\text { cancer }\end{array}$} & \multirow[t]{2}{*}{ NA } & $\begin{array}{l}\text { Semi-structured focus group (90- } \\
120 \mathrm{~min}) \text { and in-depth interview } \\
\text { (30-60 min), tape recorded }\end{array}$ \\
\hline & & & & & & & Unmet needs (pp. 191-193): \\
\hline
\end{tabular}


Table 2 Characteristics and Main Findings of the Included Studies (Continued)

Author, Year Country/ Setting Study Design Participant Diagnosis

\& QS

Region

Age (yr):

mean 75 (59-

88)

Gender: 29

(M)

\section{S49 [74]: \\ Christ G, et \\ al., 1990}

USA

QS: 1

S50 [75]:

Lam W. W.T,

Hong Kong Outpatients

ET AL., 2014

QS: 4
Outpatients Interview survey Sampling:

Sample size: 228

Age (yr): 53.4

$\pm 9.79$

Gender: 228

(F) unclear

Sample size:

200

Age (yr): $45-$

64 (54\%)

Gender: 62\%

(F)

Sampling: consecutive sampling

Advance cancer
(mixed cancer sites)

Advance breast

cancer (stage III/IV) (longitudinal)

Response
Rate


Table 2 Characteristics and Main Findings of the Included Studies (Continued)

Author, Year Country/ Setting Study Design Participant Diagnosis

\& QS Region $\quad$ Gender: $30 / 76$

ender: $30 / 76$

(F)

\begin{abstract}
S6 [32]: Park South
SM, et al., Korea
\end{abstract}

2010

QS: 1

\begin{tabular}{|c|c|c|c|c|}
\hline $\begin{array}{l}\text { S9 [35]: } \\
\text { Chen SC, et } \\
\text { al., } 2016 \\
\text { QS: } 4\end{array}$ & Taiwan & Mixed & $\begin{array}{l}\text { Questionnaire } \\
\text { survey }\end{array}$ & $\begin{array}{l}\text { Sampling: } \\
\text { consecutive } \\
\text { sampling } \\
\text { Sample size: } \\
166 \\
\text { Age (yr): } 49.6 \\
\pm 12.0 \\
\text { Gender: } 71 / \\
166 \text { (F) }\end{array}$ \\
\hline
\end{tabular}

$\begin{array}{ll}\text { survey } & \text { unclear? } \\ \text { (retrospective) } & \text { Sample size: } \\ & 1662 \\ & \text { Age (yr): not } \\ & \text { report } \\ & \text { Gender: 1099/ } \\ & 1662 \text { (F) }\end{array}$

$66(F)$
Informal caregivers cancer patients (patients died)

Age (yr): not report Gender: 1099/ $1662(\mathrm{~F})$

unclear?

Sample size: of mixed advanced
$1662 / 4042$

(41.4\%)

$\begin{array}{ll}\text { Response } & \text { Data Collection Method/ } \\ \text { Rate } & \text { Instrument \& Findings } \\ \text { information in writing" (23\%); 3) } \\ \text { "fear of an unpredictable future" } \\ \text { (22\%), 4) "difficulty in coordinating } \\ \text { the care of different professionals" } \\ \text { (22\%), 5) "difficulty in getting } \\ \text { access to help from agencies/ } \\ \text { professional organizations" (22\%); } \\ \text { 6) "difficulty in getting a second } \\ \text { opinion from another doctor" } \\ \text { (21\%), 7) "how I should handle the } \\ \text { patient's pain" (21\%), 8) "extra } \\ \text { expenditure because of the } \\ \text { disease" (17\%), 9) "insufficient } \\ \text { adjustment of hospital care to the } \\ \text { home situation" (17\%), 10) "the } \\ \text { possibility to choosing another } \\ \text { care provider" (14\%) } \\ \text { Information needs: information on } \\ \text { 1) "the physical problems" (69\%), 2) } \\ \text { "expectations for the future" (59\%), } \\ \text { 3) "the possibilities of treatment } \\ \text { and side effects" (52\%); 4) } \\ \text { "euthanasia" (41\%); 5) "cause on } \\ \text { cancer"(39\%), 6) "on nourishment" } \\ \text { (37\%); 7) "on places and agency } \\ \text { that provide help" (30\%); 8) "aids } \\ \text { to help me" (29\%) } \\ \text { Self-designed needs questionn }\end{array}$

Self-designed needs questionnaire: including 5 domains: 1) symptom management, 2) psychosocial support, 3) financial support, (4) community support, including volunteer assistance, and 5) religious support.. (p.701)

Unmet needs (p. 703): 1) symptom support (42.8\%), 2) financial. support $(42.7 \%), 3)$ psychological support $(20.6 \%)$, 4) community support (19.7\%), and 5) religious support (3.8\%)

Informal caregivers $\quad 166 / 190$

1) Partners and Caregivers supportive care needs survey (SCNS-P\&C):6 domains and 44 items

2) Numerical rating scale (NRS) (0$10,0=$ no fatigue or sleep disturbance, $10=$ worst imaginable): fatigue or sleep disturbance

Top 10 unmet needs: 1)

"Managing concerns about the cancer coming back" (78.3\%); 2) "Addressing fears about the person with cancer's physical or mental deterioration" (72.3\%); 3) "Ensuring there is an ongoing case manager to coordinate services for the person with cancer" (71.1\%); 4) "Accessing information on what the person with cancer's physical needs are likely to be" (68.7\%); 5) "Accessing information about the person with cancer's prognosis, or likely outcome" (65.1\%); 6)

"Accessing information about the 
Table 2 Characteristics and Main Findings of the Included Studies (Continued)

Author, Year Country/ Setting Study Design Participant Diagnosis

\& QS Region

Study Design Darticipant Diagnosis

Response
Rate

Data Collection Method/

Instrument \& Findings

benefits and side-effects of treatments so you can participate in decision making about the person with cancer's treatment" $(62.1 \%) ; 7)$ "Obtaining adequate pain control for the person with cancer" (61.5\%); 8) "Finding out about financial support and government benefits for you and/or the person with cancer" (60.9\%); 9) "Understanding the experience of the person with cancer" $(58.5 \%) ; 10)$ "Reducing stress in the person with cancer's life" (56.1\%)

\begin{tabular}{|c|c|c|c|c|c|c|}
\hline $\begin{array}{l}\text { S13 [9]: Cui } \\
\text { J, et al., } \\
2014 \\
\text { QS: } 4\end{array}$ & $\begin{array}{l}\text { Shanghai, } \\
\text { China }\end{array}$ & Inpatients & $\begin{array}{l}\text { Questionnaire } \\
\text { survey }\end{array}$ & $\begin{array}{l}\text { Sampling: } \\
\text { convenience } \\
\text { sampling } \\
\text { Sample size: } \\
649 \\
\text { Age (yr): } 49.2 \\
\pm 13.18 \\
\text { Gender: } 369 / \\
649 \text { (F) }\end{array}$ & $\begin{array}{l}\text { Family caregivers of } \\
\text { mixed advanced } \\
\text { cancer patients } \\
\text { (stage IV) }\end{array}$ & $\begin{array}{l}649 / 700 \\
(95.6 \%)\end{array}$ \\
\hline
\end{tabular}

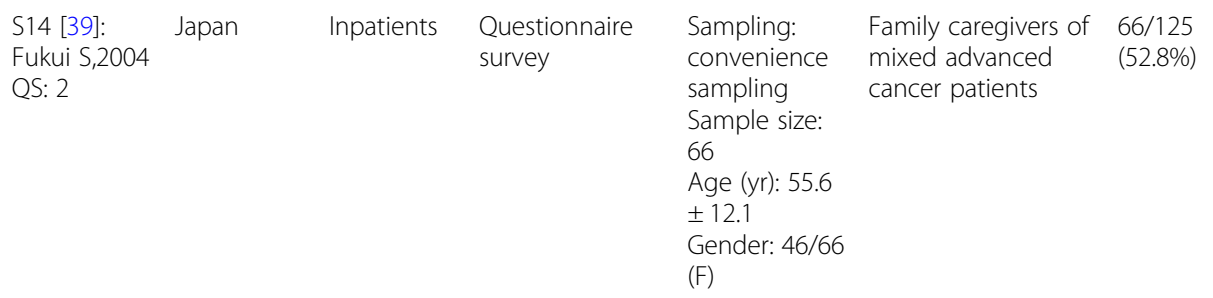

Self-designed needs questionnaire: 7 dimensions and 36 items (p. 567) Cronbach's $a=0.902$

Scores of Needs (p. 567):

1) "maintaining health" (3.48 \pm 1.04); 2) "support from professionals" (4.11 \pm 0.84$) ; 3$ ) "knowledge about disease and treatment" (4.37 \pm 0.81$)$; 4) "funeral support" (2.85 \pm 1.30$) ; 5)$ "information for hospice care" (3.01 \pm 1.14$) ; 6)$ "psychological support from patients" (3.08 \pm 1.18); 7) "symptom control for patients" (4.26 \pm 0.95$)$; 8 ) overall $(3.6 \pm 0.75)$

Self-designed information needs questionnaire: 7 items

Information needs (p. 32):

Disease-related Information

1) Information on disease (54

$82 \%)$; 2) Information on treatment $(48,73 \%) ; 3)$ Information on prognosis $(43,65 \%)$

Care-related information

1) Patients' physical care $(40,61 \%)$;

2) Patients' psychological care

(33,56\%); 3) Family care $(31,47 \%)$

\begin{tabular}{|c|c|c|c|c|}
\hline $\begin{array}{l}\text { S15 [40]: } \\
\text { Dubenske } \\
\text { LL, et al., } \\
2008 \\
\text { QS: } 3\end{array}$ & USA & NR & $\begin{array}{l}\text { Questionnaire } \\
\text { survey }\end{array}$ & $\begin{array}{l}\text { Sampling: } \\
\text { convenience } \\
\text { sampling } \\
\text { Sample size: } \\
159 \\
\text { Age (yr): } \\
50.28 \pm 12.91 \\
\text { Gender: } 159 / \\
159 \text { (F) }\end{array}$ \\
\hline
\end{tabular}

Informal female NR caregivers of mixed advanced cancer patients

Self-designed Cancer Caregiver Needs Checklist: 9 domains and 104 items

Information needs (p. 269):

1) Disease/ medical $(0.59 \pm 0.29) ; 2)$

Caregiving $(0.56 \pm 0.27)$; 3$)$ Relating with the patient $(0.59 \pm 0.31)$

4) Caregiver well-being $(0.41 \pm$ $0.30) ; 5)$ Financial/legal (0.28 \pm $0.35)$; 6$)$ Family and close others $(0.42 \pm 0.33)$

7) Future outlook (0.42 \pm 0.39$)$; 8 ) Dying $(0.48 \pm 0.33)$; 9$)$ Spirituality $(0.19 \pm 0.27)$

S24 [49]: USA NR

Mangan PA

Qualitative study Sample size: (focus group) 32

Active

caregivers ( $n$ =17)
Informal caregivers of mixed advanced cancer patients (metastasis) 
Table 2 Characteristics and Main Findings of the Included Studies (Continued)

\begin{tabular}{|c|c|c|c|c|c|}
\hline $\begin{array}{l}\text { Author, Year } \\
\& \text { QS }\end{array}$ & $\begin{array}{l}\text { Country/ } \\
\text { Region }\end{array}$ & Setting & Study Design & Participant & Diagnosis \\
\hline & & & & $\begin{array}{l}\text { Bereaved } \\
\text { caregivers ( } n \\
=15) \\
\text { Sampling: } \\
\text { unclear }\end{array}$ & \\
\hline $\begin{array}{l}\text { S26 [51]: } \\
\text { Joad ASK, et } \\
\text { al., } \\
2011 \\
\text { QS: } 2\end{array}$ & India & Mixed & $\begin{array}{l}\text { Interview survey } \\
\text { with semi- } \\
\text { structured } \\
\text { questionnaire }\end{array}$ & $\begin{array}{l}\text { Sampling: } \\
\text { unclear } \\
\text { Sample size: } \\
56 \\
\text { Age (yr): } 36 \\
\text { caregivers } \\
\text { aged 30-60 } \\
\text { Gender: } \\
\text { unclear }\end{array}$ & $\begin{array}{l}\text { Family caregivers 3- } \\
6 \text { months after the } \\
\text { death of patients } \\
\text { (cancer sites } \\
\text { unclear) }\end{array}$ \\
\hline
\end{tabular}

\begin{tabular}{|c|c|c|c|c|}
\hline $\begin{array}{l}\text { S27 [52]: } \\
\text { Buck HG, et } \\
\text { al., } 2008 \\
\text { QS: } 2\end{array}$ & USA & $\begin{array}{l}\text { Home- } \\
\text { based }\end{array}$ & $\begin{array}{l}\text { Questionnaire } \\
\text { survey }\end{array}$ & $\begin{array}{l}\text { Sampling: } \\
\text { unclear } \\
\text { Sample size: } \\
110 \\
\text { Age (yr): } 64.7 \\
\pm 14.6 \\
\text { Gender: } 83 / \\
110 \text { (F) }\end{array}$ \\
\hline
\end{tabular}

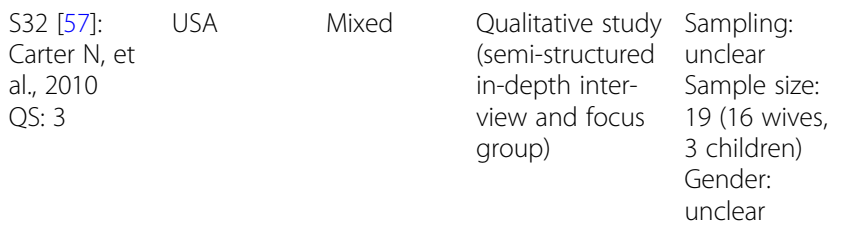

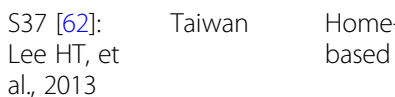

al., 2013

QS: 3

Qualitative study Sampling: (in-depth consecutive interview) sampling Sample size:
Informal caregivers NR of mixed advanced cancer patients

Family caregivers of NA advanced prostate cancer
Response Data Collection Method/

nstrument \& Finding

well-being including physical and emotional, caregivers roles); 3) help from others (practical assistance and social support) 4) unsolicited needs such as non-professional information needs, impacts on their family

Semi-structured questionnaire

Unmet needs (pp. 192-193):

1) Medical needs: "lack of home -care services" (17\%); "training in "care giving"'" (71\%); "need for an admission to a hospice/hospital" (40\%). 2) Psychological needs: 1) "felling of tense" (39\%); 2) "anxious" (17\%); 3) "depressed" (32\%); 3) Financial needs: "need financial help from other families or friends" (55.6\%); 4) Information needs:

"help in communicating disease status and prognosis with their loved one" (35\%); 5) Social needs: "lack of social life" (71.4\%);

"affected the relationships and interactions with others" (42.9\%)

Spiritual Needs Inventory (SNI): 17 items

Top 10 unmet needs of each item:

1) "be with family" (20\%); 2)

"laugh"(16\%); 3) "be with

friends" $(12 \%)$; 4) "see smiles of others"(12\%), 5) 'think happy thoughts'(11\%), 6) "be around children" (10\%); 7) "go to religious services" (10\%); 8) "talk about dayto-day things" (8\%); 9) "read inspirational materials" (8\%), 10) "talk with someone about spiritual issues" (6\%)

Semi-structured in-depth interview (40-90 min) and focus group (6090 min), audiotaped

Needs (pp. 167-168):

1) informational needs regarding disease, treatment, side effects and care services, etc.

2) "uncertainty about the future" 3) caregiver burden including supporting the physical, functional and emotions needs of patients 4) "practical assistance needs like household chores"

5) "feelings of isolation as lack of social activities" 44
Family caregivers of $44 / 49$ (89.8\%) In-depth interview with openterminal cancer patients (mixed cancer sites) (tape recorded) ended questionnaire (30-40 min)

Needs: 1) Emotional support from families and professionals including listening, encouragement, etc. 2) Information needs regarding "symptom management, nutrition, 
Table 2 Characteristics and Main Findings of the Included Studies (Continued)

\begin{tabular}{|c|c|c|c|c|c|c|}
\hline $\begin{array}{l}\text { Author, Year } \\
\& \text { QS }\end{array}$ & $\begin{array}{l}\text { Country/ } \\
\text { Region }\end{array}$ & Setting & Study Design & Participant & Diagnosis & $\begin{array}{l}\text { Respor } \\
\text { Rate }\end{array}$ \\
\hline $\begin{array}{l}\text { S39 [64]: } \\
\text { Chen HY, et } \\
\text { al.,2008 } \\
\text { QS: } 2\end{array}$ & $\begin{array}{l}\text { Shanghai, } \\
\text { China }\end{array}$ & Inpatients & $\begin{array}{l}\text { Questionnaire } \\
\text { survey }\end{array}$ & $\begin{array}{l}\text { Sampling: } \\
\text { convenience } \\
\text { sampling } \\
\text { Sample size: } \\
89 \\
\text { Age (yr): (23- } \\
\text { 72, median } \\
\text { 52.1) } \\
\text { Gender: 58/89 } \\
\text { (F) }\end{array}$ & $\begin{array}{l}\text { Family caregivers of } \\
\text { advanced cancer } \\
\text { patients (cancer } \\
\text { sites unclear) }\end{array}$ & $\begin{array}{l}89 / 100 \\
(89.0 \%)\end{array}$ \\
\hline
\end{tabular}

\author{
Instrument \& Findings
}

concerns about dying, medication and nursing aids" (p. 633).

Self-designed questionnaire (unclear items)

Needs (p. 19):

1) prognosis of disease $(100 \%)$; 2 )

help to realize patient's

wishes(100\%); 3) continuous support after discharge from hospital(100\%); 4) knowledge of self-care(100\%); 5) relevant knowledge of disease $(98.9 \%)$; 6) regular counseling service (84.3\%); 7) emotional support(69.7\%); 8) pain management of patients(59.6\%); 9) accompany (50.6\%)

Studies Regarding Both Advanced Cancer Patients and their Informal Caregivers $(n=5)$

\begin{tabular}{|c|c|c|c|c|c|}
\hline $\begin{array}{l}\text { Author, Year } \\
\& \text { QS }\end{array}$ & Region & Setting & Study Design & Participants & Diagnosis \\
\hline $\begin{array}{l}\text { S23 [48]: } \\
\text { Dehghan R, } \\
\text { et al., } \\
2012 \\
\text { QS: } 4\end{array}$ & Bangladesh & Outpatients & $\begin{array}{l}\text { Qualitative study } \\
\text { (in-depth } \\
\text { interview) }\end{array}$ & $\begin{array}{l}\text { Sampling: } \\
\text { convenience } \\
\text { sampling } \\
\text { Sample size: } \\
20 \\
\text { Patients ( } n= \\
\text { 3), Family } \\
\text { members ( } n \\
=9) \text {, Clinical } \\
\text { staffs }(n=8)\end{array}$ & $\begin{array}{l}\text { Advanced breast } \\
\text { cancer and family } \\
\text { members }\end{array}$ \\
\hline
\end{tabular}

\section{Response Data Collection Method/ Rate Instrument \& Findings}

NA

Semi-structured in-depth interview with open-ended questions (tape recorded) and qualitative description for analysis

Needs (pp. 147-148): 1) "social needs of patients and families" due to financial impact, economic uncertainty and needs for social security;

2) "psychological and spiritual needs of patients and families": feeling of sadness, anxiety, anger, abandonment, fear and hopeless; 3) "need for information among patients and families".

4) "Access to and receipt of care from professional systems and providers"

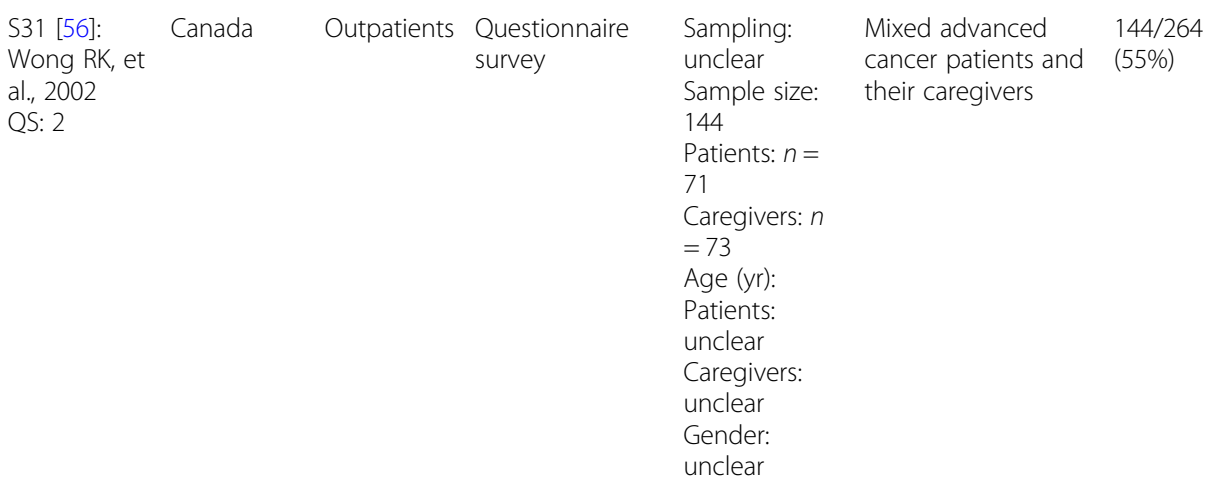

\begin{tabular}{|c|c|c|c|c|c|}
\hline $\begin{array}{l}\text { S34 [59]: } \\
\text { Hwang SS, } \\
\text { et al., } 2003 \\
\text { QS: } 4\end{array}$ & USA & Mixed & $\begin{array}{l}\text { Questionnaire } \\
\text { survey }\end{array}$ & $\begin{array}{l}\text { Sampling: } \\
\text { consecutive } \\
\text { sampling } \\
\text { Sample size: } \\
100\end{array}$ & $\begin{array}{l}\text { Informal caregivers } \\
\text { of advanced cancer } \\
\text { patients (cancer } \\
\text { sites unclear) }\end{array}$ \\
\hline
\end{tabular}

Advanced Cancer Information Needs Survey (ACIN): 22 items

Needs for patients:

1) "pain control" (75\%), 2)

"weakness and fatigue" (58\%), 3)

"shortness of breath" (52\%), 4)

"what cause cancer" (48\%), 5)

"home care services" (46\%), 6)

"communicating with loved ones" (46\%)

Needs for caregivers:

1) "pain control" (82\%), 2)

"weakness and fatigue" (66\%), 3)

"home care services" (58\%), 3) "what cause cancer" (53\%), 4) "how can we prevent cancer" (58\%), 5)

"why are some cancers not curable" (56\%)

The Family Inventory of Needs (FIN): 20 items

Caregiver's Perception of Patients' Unmet Needs (PPUN): 14 items

Perception of Patients' Unmet Needs (PPUN): 
Table 2 Characteristics and Main Findings of the Included Studies (Continued)

\begin{tabular}{|c|c|c|c|c|c|c|c|}
\hline $\begin{array}{l}\text { Author, Year } \\
\& \text { QS }\end{array}$ & $\begin{array}{l}\text { Country/ } \\
\text { Region }\end{array}$ & Setting & Study Design & Participant & Diagnosis & $\begin{array}{l}\text { Response } \\
\text { Rate }\end{array}$ & $\begin{array}{l}\text { Data Collection Method/ } \\
\text { Instrument \& Findings }\end{array}$ \\
\hline & & & & $\begin{array}{l}\text { Age (yr): (27- } \\
\text { 85, median } \\
62 \text { ) } \\
\text { Gender: } \\
\text { unclear }\end{array}$ & & & $\begin{array}{l}\text { 1) physical ( } 80 \%), 2 \text { ) nutritional } \\
(51 \%), 3 \text { ) daily living }(44 \%), 4) \\
\text { emotional (33\%). } \\
\text { Caregiver unmet needs (FIN): } \\
\text { 1) "having information about what } \\
\text { to do for the patient at home" } \\
\text { (37\%); } 2 \text { ) "knowing when to expect } \\
\text { symptoms to occur" (31\%); } 3 \text { ) } \\
\text { "being told about people who } \\
\text { could help with problems" (26\%); } \\
\text { 4) "knowing the probable outcome } \\
\text { of the patient's illness" (26\%) }\end{array}$ \\
\hline \multirow[t]{2}{*}{$\begin{array}{l}\text { S38 [63]: } \\
\text { Liu Y, } 2008 \\
\text { QS: } 3\end{array}$} & \multirow[t]{2}{*}{$\begin{array}{l}\text { Shanghai, } \\
\text { China }\end{array}$} & \multirow[t]{2}{*}{$\begin{array}{l}\text { Home- } \\
\text { based }\end{array}$} & \multirow[t]{2}{*}{$\begin{array}{l}\text { Questionnaire } \\
\text { survey }\end{array}$} & \multirow{2}{*}{$\begin{array}{l}\text { Sampling: } \\
\text { convenience } \\
\text { sampling } \\
\text { Sample size: } \\
400 \\
\text { Age (yr): } \\
\text { Patients:60.61 } \\
\pm 12.67 \\
\text { Caregivers: } \\
56.04 \pm 12.57 \\
\text { Gender: } \\
\text { Patients:63/ } \\
115(F) \\
\text { Caregivers:29/ } \\
113(F)\end{array}$} & \multirow{2}{*}{$\begin{array}{l}\text { Mixed cancer } \\
\text { patients at stage III/ } \\
\text { IV and their } \\
\text { caregivers }\end{array}$} & \multirow{2}{*}{$\begin{array}{l}228 / 400 \\
(57 \%) \\
\text { (patients:115, } \\
\text { caregiver:113) }\end{array}$} & $\begin{array}{l}\text { Self-designed needs questionnaire } \\
\text { for advanced cancer patients and } \\
\text { their caregivers }\end{array}$ \\
\hline & & & & & & & $\begin{array}{l}\text { Needs for patients (pp. 30-31): } \\
\text { 1) psychological: families' } \\
\text { understanding and support(96.5\%), } \\
\text { etc. 2) Physical care: information of } \\
\text { treatment, rehabilitation ( } 80.9 \%), \\
\text { etc. 3) Social: peer activities and } \\
\text { support (54.8\%), etc. } \\
\text { Needs for caregivers (p. 38): } \\
\text { 1) psychological: communication } \\
\text { with families and professionals } \\
\text { (76.1\%), etc. 2) social: information } \\
\text { about treatment and } \\
\text { prognosis( } 81.4 \%) \text { etc. } 3) \\
\text { educational: medication } \\
\text { guidance(80.5\%) etc. }\end{array}$ \\
\hline \multirow{2}{*}{$\begin{array}{l}\text { S42 [67]: } \\
\text { Miu J, et al., } \\
2016 \\
\text { QS: } 2\end{array}$} & \multirow[t]{2}{*}{$\begin{array}{l}\text { Shanghai, } \\
\text { China }\end{array}$} & \multirow[t]{2}{*}{ Inpatients } & \multirow[t]{2}{*}{$\begin{array}{l}\text { Questionnaire } \\
\text { survey }\end{array}$} & \multirow[b]{2}{*}{$\begin{array}{l}\text { Sampling: } \\
\text { unclear } \\
\text { Sample size: } \\
42 \text { (42 } \\
\text { patients and } \\
42 \text { family } \\
\text { caregivers) } \\
\text { Age (yr): } \\
\text { Patients:72.9 } \\
\pm 11.6 \\
\text { Caregivers: } \\
55.9 \pm 13.45 \\
\text { Gender: } \\
\text { Patients:18/42 } \\
\text { (F) } \\
\text { Caregivers:23/ } \\
42 \text { (F) }\end{array}$} & \multirow[t]{2}{*}{$\begin{array}{l}\text { Mixed advanced } \\
\text { cancer patients and } \\
\text { their caregivers }\end{array}$} & \multirow[t]{2}{*}{ 42/45 (93.3\%) } & $\begin{array}{l}\text { Self-designed needs questionnaire } \\
\text { for advanced cancer patients and } \\
\text { their caregivers [63] }\end{array}$ \\
\hline & & & & & & & $\begin{array}{l}\text { Needs for patients (p. 2387): } \\
\text { 1) "families' understanding and } \\
\text { support" (2.43 } \pm 0.59) ; 2) \text { "relieving } \\
\text { constipation" (2.38 } \pm 0.62) \\
\text { 3) "psychological support for } \\
\text { caregivers after the death of } \\
\text { themselves" (2.36 } \pm 0.66) ; 4) \text { "pain } \\
\text { assessment" (2.33 } \pm 0.61) ; 5) \text { "pain } \\
\text { management" (2.31 } \pm 0.64) ; 6) \\
\text { "improving appetite" (2.31 } \pm 0.6) \\
\text { Needs for caregivers: } \\
\text { 1) "dietary and nutrition" (2.38 } \pm \\
0.66) ; 2) \text { "guidance about how to } \\
\text { help patients do activities" (2.38 } \pm \\
0.66) ; 3) \text { "pain assessment" (2.38 } \pm \\
0.73) ; 4) \text { "communication between } \\
\text { families and professionals" (2.36 } \pm \\
0.58) ; 5) \text { "information about } \\
\text { treatment and prognosis" (2.33 } \pm \\
0.65)\end{array}$ \\
\hline
\end{tabular}

Notes 1: QS: overall quality score; ADL: Activities of daily living; M: male; F: female; G1: group1; G2: group2; G3: group3; EC: Esophageal; PBC: Pancreaticobiliary; EORTC QLQ-OES18: EORTC QLQ-Esophagus (OES) 18 (Esophagus cancer module) questionnaire; EORTC QLQ-PAN26: EORTC QLQ-Pancreatic (PAN) 26 (Pancreatic cancer module) questionnaire; EORTC QLQ-C30: European Organization for Research and Treatment of Cancer Quality of Life Core 30; a: only the baseline data was used in this review

Notes 2: in the "Data Collection Method/ Instrument \& Findings" column, direct quotations from several included quantitative studies using commonly utilized research scales with documented psychometric properties were details of each of the used research questionnaire items. Thus, information regarding page numbers was not provided, but that for direct quotations from studies using self-designed semi-structured questionnaires and/or qualitative methods, as well as page numbers for such quotations, was provided 
Table 3 Overall unmet needs domains and prevalence ranges of prominent items by each domain (Patients)

\begin{tabular}{|c|c|c|c|}
\hline Domains & $\begin{array}{l}\text { Number of } \\
\text { studies }\end{array}$ & Subdomains/ items & Prevalence ranges \\
\hline \multirow[t]{7}{*}{ Physical } & \multirow[t]{7}{*}{22} & Fatigue & $\begin{array}{l}18-76.3 \%[6,31,33,34,42,43,45,47,51,54 \\
56,63]\end{array}$ \\
\hline & & Pain & $18-75 \%[6,31,33,36,45,47,50,60,66]$ \\
\hline & & Sleep problems & $21.1-37.1 \%[28,47]$ \\
\hline & & Dyspnea & $19-67.3 \%[36,45,56]$ \\
\hline & & Lack of appetite & $13-80 \%[45,50,55,63]$ \\
\hline & & gastrointestinal symptoms & $12-45.1 \%[45,66]$ \\
\hline & & "Felling unwell a lot of the time" & $17.3-44.7 \%[6,33,50]$ \\
\hline \multirow{2}{*}{$\begin{array}{l}\text { Activities of Daily Living } \\
\text { (ADL) }\end{array}$} & \multirow[t]{2}{*}{11} & "not being able to do the things you used to do" & $19-46.9 \%[6,33,50,58,60]$ \\
\hline & & "Work around the home" & $18.6-44.2 \%[6,33,50,55]$ \\
\hline \multirow[t]{9}{*}{ Psychological } & \multirow[t]{9}{*}{25} & "Uncertainty about the future" & $21.4-62.4 \%[6,31,33,41,60]$ \\
\hline & & Emotional Support & $\begin{array}{l}10.1-84.4 \%[6,28,29,31,33,36,41,45,46,50, \\
70,72,73] \\
\text { (Anxiety [6, 33]: 15.3-41.8\%; Depression [31, } \\
33,41,50]: 15-62.4 \% \text { ) }\end{array}$ \\
\hline & & $\begin{array}{l}\text { "worry that the results of treatment are beyond your } \\
\text { control" }\end{array}$ & $19-71.8 \%[6,41,50,58,60]$ \\
\hline & & "Feeling about death and dying" & $32.5-62.4 \%[33,41]$ \\
\hline & & "Fears about the cancer spreading" & $17.6-78.8 \%[6,31,41,42,47,50,55]$ \\
\hline & & "concerns about the worries of those close to you" & $27.9-68.2 \%[6,33,41,50,60]$ \\
\hline & & "Support in coping" & $24.3-57.5 \%[29,54,55]$ \\
\hline & & "Learning to feel in control of your situation" & $32.5-56.5 \%[33,41]$ \\
\hline & & "Fear of physical suffering" & $16.7-62.9 \%[31,34,36,47,50]$ \\
\hline \multirow[t]{2}{*}{ Social } & \multirow[t]{2}{*}{9} & family and friends' support & $9.9-96.5 \%[29,45,54,55,63,65,67]$ \\
\hline & & volunteers & $18.7 \%[65]$ \\
\hline Communication & 5 & Communication & $7.7-87.9 \%[28,29,56,63,66]$ \\
\hline Financial & 8 & Financial & $6.6-72 \%[28,31,36,43,46,54,55,70]$ \\
\hline \multirow[t]{3}{*}{ Spiritual } & \multirow[t]{3}{*}{5} & Meaning of death & $15-85.4 \%[31,36]$ \\
\hline & & Religious & $44 \%[43]$ \\
\hline & & "being able to choose the place where you want to die" & $11-15 \%[54,55]$ \\
\hline \multirow[t]{2}{*}{ Autonomy } & \multirow[t]{2}{*}{5} & "I can do less than before" & $17-83 \%[31,34,43]$ \\
\hline & & "experiencing loss of control over one's life" & $16-19 \%[31,47]$ \\
\hline \multirow[t]{2}{*}{$\begin{array}{l}\text { Patients care and } \\
\text { support }\end{array}$} & \multirow[t]{2}{*}{3} & $\begin{array}{l}\text { "Reassurance by medical staff that the way you feel is } \\
\text { normal" }\end{array}$ & $32.5-56.5 \%[33,41]$ \\
\hline & & $\begin{array}{l}\text { "doctor acknowledges and shows sensitivity to your } \\
\text { feelings and emotional needs" }\end{array}$ & $34.8-39.5 \%[33,42]$ \\
\hline \multirow[t]{7}{*}{$\begin{array}{l}\text { Healthcare service and } \\
\text { information }\end{array}$} & \multirow[t]{7}{*}{14} & $\begin{array}{l}\text { "Being informed about things you can do to help yourself } \\
\text { to get well" }\end{array}$ & $41-65.9 \%[33,41,42,60,75]$ \\
\hline & & $\begin{array}{l}\text { "Having one member of hospital staff with whom you can } \\
\text { talk to" }\end{array}$ & $32-72 \%[33,41,75,58,75]$ \\
\hline & & "Being informed about your test results as soon as feasible" & $50.8-62.5 \%[41,42,75]$ \\
\hline & & "benefit and side-effects of treatment" & $4-66.7 \%[31,41,42,44,54,63,66,69,75]$ \\
\hline & & $\begin{array}{l}\text { "Being given written information about the important } \\
\text { aspects of your care" }\end{array}$ & $42.3-52.9 \%[41,75]$ \\
\hline & & "Being treated like a person not just another case" & $34.5-54.1 \%[37,41,61,75]$ \\
\hline & & "Being informed about cancer which is under control" & $54.1-60.4 \%[41,75]$ \\
\hline Sexuality & 4 & Sexuality & $5-75 \%[31,36,45,58]$ \\
\hline
\end{tabular}


Table 3 Overall unmet needs domains and prevalence ranges of prominent items by each domain (Patients) (Continued)

\begin{tabular}{lcll}
\hline Domains & $\begin{array}{c}\text { Number of } \\
\text { studies }\end{array}$ & Subdomains/ items & \multicolumn{1}{c}{ Prevalence ranges } \\
\hline Nutrition & 2 & Nutrition & $38.9-43.2 \%[28,66]$ \\
Counseling & 1 & & $17-24 \%[31]$ \\
\hline
\end{tabular}

Notes: Needs items (sentences or phrases) which were put in the quotation marks were directly extracted from the corresponding included studies

from professional staff” (information needs) [48, 72, 73], "need more social security" (social needs) [48], and "not being regarded as a person" (p. 178) (healthcare service and information needs) [72]. However, the needs in qualitative studies were more detailed than those in quantitative studies, and the specific causes of unmet needs were identified. For example, patients elaborated that "lack of dialogue with the professionals led some patients to feel neglected and uncertain in their sense of belonging" (p. 178) [72] was the cause of "not being regarded as a person" (p. 178). Additionally, "sadness, anger, frustration and regret" resulted from "some unsolved issues about diagnosis and treatment decisions" [73]. Several unmet needs identified from the qualitative data were not identified in quantitative studies. For instance, subjects expressed "what they had achieved in their lives and what still needed to be done before death" (p. 42), "establish themselves as they 'really' are" (p. 41) (spiritual needs) [71], and "patients want to be proactive in problem solving" (p. 179), but they did not know how to do it (autonomy needs) [72].

\section{Descriptions of unmet needs in informal caregivers}

Seven unmet need domains were extracted on the basis of qualitative $(n=4)$ and quantitative $(n=13)$ studies.

In terms of the quantitative studies, the sample size ranged from 42 to 1662 , with the mean sample size being 259. The response rates ranged from 41.4 to $95.6 \%$. Seven domains, including information, physical, psychological, financial, cancer care service, spiritual, and social needs, were identified. Information domain included two subdomains, namely, illness and treatment and care-related information. Unmet needs regarding illness and treatment information were mentioned in nine studies, and the prevalence ranged from 26 to $100 \%$ [9, 30, 35, 39, 40, 51, 56, 67, 63]. Care-related information was reported in 10 studies with the prevalence rate in the range of $21-100 \%[9,30,35$, $39,40,51,59,63,64,67]$. With regard to cancer care services, $21-72.3 \%$ of the informal caregivers presented unmet needs in terms of quality of care $[29,30,35]$, and $14-100 \%$ reported unmet needs on transitional care services [30, 32, $51,64]$. The percentages of the five other domains, including physical, psychological, financial, spiritual, and social unmet needs, were $42.8 \%$ [32], 17-78.3\% [32, 35, 51, 63, 64], 17-67.3\% [30, 32, 35, 51], 3.8-100\% [30, 32, 52, 64], and $42.9-71.4 \%$ [51], respectively. Furthermore, "managing concerns about the cancer coming back" (78.3\%) [35], "finding out about financial support and government benefits for you and/or the person with cancer" (60.9\%) [35], "help to realize patient's wishes" (100\%) [64], and "lack of social life" (71.4\%) [51] were reported as the most common psychological, financial, spiritual, and social needs.

According to four qualitative studies [48, 49, 57, 62], three similar unmet need domains, namely, informational, psychological, and social needs, were identified through summative content analysis. Informal caregivers commonly stated about "unmet information needs in terms of disease, treatment, side effects, care services, symptom management, nutrition, medication and nursing aids" (informational) [48, 57, 62], "feelings of sadness and loneliness, as well as a sense of abandonment, fear and helplessness" (p. 147) [48] or "insufficient listening and encouragement from other family members and professionals" [62] (psychological), and "feelings of isolation due to the lack of social activities" (social) [57]. Several specific unmet needs, including the manner of communication between professional staff and caregivers or patients, the administration and function of the healthcare system, and some practical assistance, such as cleaning the house and walking the dog [49], were also identified in qualitative studies [49].

\section{Variables associated with the unmet needs of patients with advanced cancer}

Variables associated with the unmet needs of patients with advanced cancer are summarized in Table 4. Relevant variables were categorized as patient-related variables (demographics, disease-related, physical, and psychological) and informal caregiver-related variables (age, gender, and psychological distress of informal caregivers).

In several studies, age, gender, marital status, education level, and income level were insignificantly associated with patients' unmet needs. Although a significant relationship was reported, results were inconsistent across studies in terms of age and marital status. With regards to gender, three studies $[28,33,63]$ reported that female patients indicated more physical and psychological unmet needs than those of male patients. Patients who were living alone experienced high psychological needs [28], and patients with high educational level presented considerable unmet needs in physical [42], ADL [42], information [44], community service [46], and sexuality [58] domains. Moreover, financial needs were less reported in patients with high income $[46,63]$. 


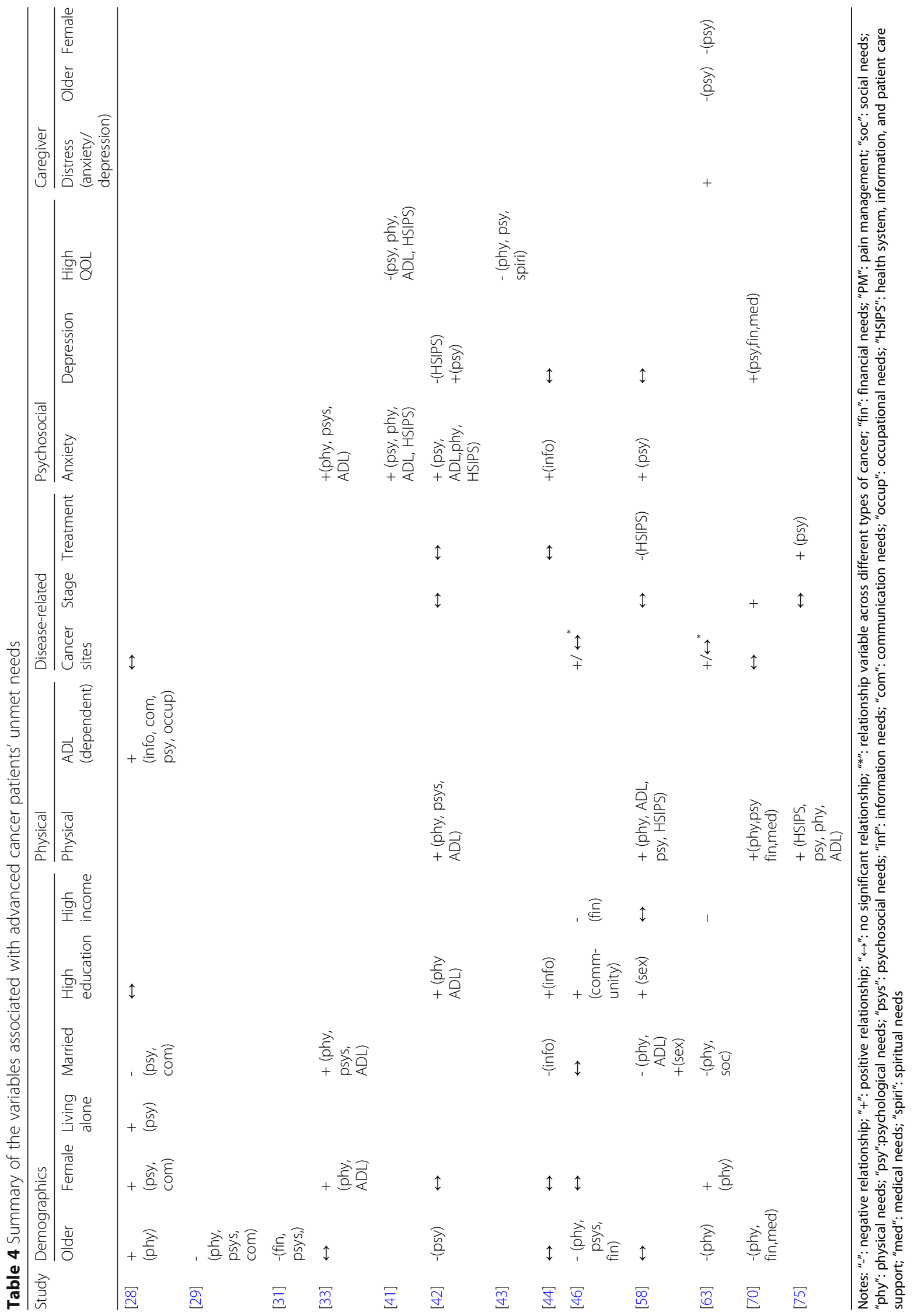


Four studies [42, 58, 70, 75] explored the relationships between symptom distress and unmet needs, and all these studies showed that patients with symptom distress experienced more unmet needs in the psychological, physical, and ADL domains. Patients with poor ability in daily living [28] indicated more unmet needs than those of independent patients, especially in terms of information, communication, psychological, and occupational needs.

Two studies $[28,70]$ showed that no relationships were observed between cancer site and their unmet needs, but two other $[46,63]$ studies showed opposite results. Two $[42,75]$ out of five studies reported that no relationship was observed between cancer stage (only stages III and IV) and unmet needs, and three ones $[58,63,70]$ indicated that patients with stage IV cancer presented more unmet needs than those with stage III cancer. Results were inconsistent across studies for cancer treatment, with two studies showing no relationship [42, 44] and two other studies suggesting either positive [75] or negative [58] relationship.

Patients with anxiety experienced high levels of physical, psychological, healthcare, and information, as well as ADL unmet needs, which was confirmed across several studies [33, 41, 42, 44, 58]. Patients with depression [42, 44, 58, 70] demonstrated varied results. Patients with low quality of life showed high unmet needs, especially in physical and psychological domains [41, 43]. Patients reported more unmet needs when their caregivers were male [28], young people [28], or those who suffered from psychological distress [28].

\section{Variables associated with the unmet needs of informal caregivers}

Older caregivers [30, 35] showed less unmet needs in terms of financial, social, and care-related information needs than those of younger caregivers. Caregivers in different caregiving settings reported different levels of unmet needs (home>general hospital>hospice care unit) $[32,39]$. Caregivers with many physical problems experienced many unmet needs [35, 63]. Caregivers had higher levels of unmet needs when patients suffered from anxiety [35], depression [35], or low physical performance [35]. Results varied across studies in terms of gender [30], length of caregiving $[9,63]$, and education level of caregivers [63] (Table 5). Similarly, results were conflicting with regard to the relationships between caregivers and patients. One study [39] showed that spousal caregivers presented many information needs, and another study [63] indicated that non-spousal caregivers reported many unmet needs.

\section{How their unmet needs were assessed in the included studies}

For patients with advanced cancer, the most commonly used multidimensional instruments were Supportive Care Needs Survey (SCNS, $n=8$ ) $[6,33,41,50,58,60,61$, 75], Problems and Needs in Palliative Care questionnaire (PNPC, $n=5$ ) [31, 34, 36, 44, 47], and Needs Assessment of Advanced Cancer Patients (NA-ACP, $n=3$ ) [38, 54, 55]. Other multidimensional instruments that were adopted included Cancer Needs Questionnaire [42], Patient Needs Assessment in Palliative Care [43], 3-Levels-of-Needs Questionnaire [45], Needs Assessment Tool: Progressive Disease-Cancer [68], Caregiver's Perception of Patients' Unmet Needs [59], and other instruments without reporting their psychometric properties. Among studies that focused on one specific need domain $(n=4)$, three explored information needs $[44,56,69]$, and one investigated spiritual needs [37]. The unidimensional

Table 5 Summary of the variables associated with informal caregivers' unmet needs

\begin{tabular}{|c|c|c|c|c|c|c|c|c|c|c|}
\hline \multirow[t]{2}{*}{ Study } & \multicolumn{5}{|c|}{ Demographics of caregivers } & \multirow{2}{*}{$\begin{array}{l}\text { Caregivers' } \\
\text { physical } \\
\text { symptom }\end{array}$} & \multirow{2}{*}{$\begin{array}{l}\text { Relationship } \\
\text { Spousal } \\
\text { caregivers }\end{array}$} & \multicolumn{3}{|c|}{ Patients-related } \\
\hline & Older & Female & $\begin{array}{l}\text { Education } \\
\text { level }\end{array}$ & $\begin{array}{l}\text { Length of } \\
\text { caregiving }\end{array}$ & Care setting & & & $\begin{array}{l}\text { Patients' } \\
\text { anxiety }\end{array}$ & $\begin{array}{l}\text { Patients' } \\
\text { depression }\end{array}$ & $\begin{array}{l}\text { Lower } \\
\text { physical } \\
\text { performance }\end{array}$ \\
\hline [30] & $\begin{array}{l}-(\text { fin, } P M, \\
\text { soc.,) }\end{array}$ & $\begin{array}{l}\text { F } \\
\text { (+phy) } \\
M(+ \\
\text { inf })\end{array}$ & & & & & & & & \\
\hline [32] & & & & & $\begin{array}{l}\text { Conventional hospital care > } \\
\text { hospice care (symptom } \\
\text { management, psy support, } \\
\text { religious support) }\end{array}$ & & & & & \\
\hline [35] & & & & & & + (overall) & & $+($ overall) & +(overall) & + (overall) \\
\hline [9] & & & & - & & & & & & \\
\hline [39] & - & & & & Home $>$ hospital (inf) & & + & & & \\
\hline [63] & $-($ soc,psy,inf $)$ & & $\begin{array}{l}-(p s y) \\
+(\text { soc })\end{array}$ & $+(\mathrm{sOc})$ & & + & -(inf) & & & \\
\hline
\end{tabular}

Notes: "-": negative relationship; "+": positive relationship; "fin": financial needs; "PM": pain management; "soc": social needs; "phy": physical needs; "inf": information needs; "overall": overall needs 
instruments adopted included the following: Toronto Information Needs Questionnaire [69], Advanced Cancer Information Needs [56], PNPC (only used the items of the information domain) [44], and an instrument [37] for spiritual needs assessment without specifying its psychometric properties. Overall, more than half of the quantitative studies (20/34) adopted instruments with acceptable validity and reliability.

Among the 13 quantitative studies reporting unmet needs of informal caregivers, comprehensive unmet needs (multiple domains) were explored in 10 studies $[9,30,32,35,40,51,59,63,64,67]$. Different quantitative studies used different measures, which included PNPC questionnaire-caregiver form [30], Family Inventory of Needs [59], Partners and Caregivers SCNS [35], needs of family caregivers of patients with advanced cancer [9], and other self-designed instruments [32, 40, 51, 63, 64, 67]. Among the three other studies that focused on unidimensional needs assessment, two $[39,56]$ measured information needs, and one [52] explored spiritual needs. The scales used were Spiritual Needs Inventory [52] and two other self-designed instruments, namely, with [56] or without [39] psychometric property testing. Among all the 13 studies, only four studies used scales with documented psychometric properties.

\section{Discussion}

The included studies highlighted that both advanced cancer patients and their informal caregivers possess a wide range of unmet needs. Psychological and physical unmet needs are two areas of focus for patients with advanced cancer; this result is consistent with a previously published review [7]. Among informal caregivers who had experience in managing patients' negative emotions, more than $30 \%$ of them reported that emotional management is the most challenging part of caregiving [76]. Three other unmet needs, namely, the need for autonomy, communication, and nutrition, were identified in this review compared with the previous review [7]. These needs may be related to the differences in cultural contexts, healthcare systems, and economic levels because several included studies in this review were conducted in eastern and developing countries. For instance, the need for autonomy is commonly culture-related [36]. Family members usually make decisions for patients in eastern cultures because family-collective decision-making is much more popular there than in other cultures [77]. This result showed the importance of developing tailored healthcare services or interventions based on context-specific unmet needs. Disease-related information needs were the most commonly reported unmet needs of informal caregivers. Considerably fewer studies reported unmet needs that are associated with the caregivers' own well-being, as they generally focus more on the patients' well-being than their own [30]. The prominent care needs of each domain were identified for patients with advanced cancer and informal caregivers in this review provide useful information and evidence for the development and implementation of tailored healthcare services. For example, emotional support was identified as the most commonly unmet need in the psychological domain for patients, thereby indicating that emotional distress (e.g., anxiety and depression) management should be a priority when providing mental health services. In addition, patients with advanced cancer and informal caregivers' unmet need domains involved multiple disciplines, which indicated that healthcare services should be multidisciplinary. The value of multidisciplinary care for patients with cancer has been well recognized [78]. Support for informal caregivers is suboptimal in many instances [79]. The unmet needs of informal caregivers are often ignored and excluded from healthcare planning [80, 81].

The prevalence of unmet needs varied across the quantitative studies for both patients and caregivers. This variability may be caused by the heterogeneity of the included studies, which were conducted within different cultural contexts, healthcare systems, and economic levels that may be associated with unmet needs. High-income countries or regions generally present well-established healthcare service systems, which can facilitate the timely identification and resolution of healthcare problems (several physical symptoms particularly require high-quality professional support [28]). Different study designs, especially the diverse instruments used, for unmet needs assessment also contribute to this heterogeneity. The highlighted heterogeneity makes it difficult to gauge and pool the percentages of unmet needs by domains. SCNS was the most commonly used instrument, which was used in eight studies. However, these eight studies adopted five different variants of the same scale, with 13 [61], 33 [58], 34 [6, 33, 41], 59 [60], and 61 items [50] for each of the five versions. Different methods of need classification are also a major barrier in gauging unmet needs by domains. For instance, in SCNS, several items were classified as spiritual needs (e.g., [50]). In other studies, the same items were coded as psychological needs (e.g., [41]). Moreover, approaches in defining unmet needs were inconsistent. Among studies that utilized the SCNS, several of them regarded moderate and high levels of need as unmet needs (e.g., [41]. In other studies, low need level was calculated as an unmet need (e.g., [50]). Different reporting methods also caused heterogeneity. Several studies reported the prevalence of unmet needs by domains without specifying the percentage of items within each domain. Some studies (e.g., [33]) only listed the prevalence of the top 10 or 20 items without reporting the prevalence by domain. Thus, directly 
combining the prevalence of reported items within a domain may increase the risk of overestimating the actual unmet need level [21].

Although consistent results across studies showed that patients with advanced cancer with symptoms of distress and anxiety and low quality of life are more likely to report high demands of unmet needs, the conclusion must be interpreted with caution. Causality cannot be established because almost all of the included studies were cross-section in design. Other patient-related variables with inconsistent results, (e.g., gender, marital status, education level, cancer site, and depression) may be caused by cultural differences and/or methodological flaws (e.g., insufficient sample size to explore relationships between two factors) of the included studies. Hence, more longitudinal studies with rigorous study designs should be adopted. In addition, whether caregivers' health outcomes were associated with the unmet needs of patients is still unclear because of the limited evidence that can be drawn from current studies. Therefore, more studies should focus on caregiver-related variables. Relevant studies regarding variables associated with informal caregivers' unmet needs are limited, and no conclusion can be drawn from the current findings.

Patients with cancer at an advanced stage commonly experience fluctuating unmet needs over time due to rapid disease progression [6]. Nevertheless, little is known about how patients with advanced cancer and/or their informal caregivers' unmet needs change across the illness trajectory. Almost all the included quantitative studies investigated unmet needs at a single time point with cross-sectional study designs. Unmet care needs assessment in the majority of the included studies is also mainly problem-oriented from a biomedical lens. Few studies considered contextual issues (sociocultural and healthcare service provisions) when assessing and interpreting results in a given context although it will be of benefit to the development and implementation of tailored interventions at a local level. Accordingly, qualitative studies are an appropriate approach because it can explore participants' in-depth experience and subjective feelings that cannot be measured by quantitative methods; additionally, the scope can be much broader than those of quantitative methods [82, 83]. Deeper understanding of unmet needs can be extracted from the qualitative studies than from quantitative findings. However, limited studies adopted qualitative study designs, and only few studies utilized mixed methods. Care needs should be comprehensively evaluated from all stakeholders, including patients, caregivers, and healthcare providers [84]. A comprehensive understanding of both patients with advanced cancer and informal caregivers' unmet needs can enable healthcare providers to develop evidence-based and tailored interventions
[18]. Nevertheless, the majority of the included studies assessed patients' unmet needs only, and almost all included studies examined unmet needs from the participants' own perspective rather than from the perspectives of all relevant stakeholders. Despite that the concept of patient-and-family-centered care is advocated by the WHO [16], structured unmet needs assessment of informal caregivers is still an uncommon practice. Only a few studies assessed the unmet needs of patients and informal caregivers, and their unmet needs were assessed separately. The mechanism of integrating the data of patients and caregivers should be considered to further embody the conceptualization as a whole unit. Focused group with mixed samples, including patients and informal caregivers in the same group, may be an appropriate approach. Finally, research instruments used for needs assessment in several included studies were inappropriate. Some scales are generic ones used for supportive care needs assessment. Several items, such as "fear about the cancer spreading," may be unsuitable for patients with advanced cancer.

A strength of this systematic review is that a large number of studies with considerable information were assimilated and analyzed through a systematic method, which can minimise biases and facilitate reliable conclusions. This work is the first systematic review conducted by considering patients with advanced cancer and their informal caregivers as a whole unit. However, this review also presents several limitations. First, subgroup analysis in terms of contexts and economic levels was not conducted. Second, given the confounding factors and insufficient number of studies in each subgroup, meta-analysis was also not performed to compare the prevalence of each identified need domain. Third, language bias cannot be excluded because only papers that were published in English or Chinese language were included. Finally, instruments for needs assessments were only summarized from the included studies, and studies in terms of instrument development were excluded.

\section{Conclusions}

A wide range of unmet care needs existed in both advanced cancer patients and informal caregivers. Given the context-bound feature, their unmet needs should be comprehensively assessed and interpreted from the perspectives of all stakeholders within a given context by using rigorous mixed methods research and longitudinal research with prospective study designs. Assessing unmet care needs by viewing patients with advanced cancer and their informal caregivers as a whole unit is highly desirable. Associated factors of their unmet needs should not be ignored, which can provide evidence for decision-making with regards to healthcare resource allocation. The value of better examining unmet needs 
and their associated factors in advanced cancer patients and informal caregivers ultimately depends on how well it could inform the development and implementation of tailored healthcare service or intervention.

\section{Endnotes}

${ }^{1}$ In this review, patients with advanced cancer are defined as follows: patients with advanced, secondary, metastatic, or terminal cancer $[23,24]$ or patients with cancer at stage III or IV according to TNM Staging System or Dukes' D according to Dukes' staging system [23, 24]).

${ }^{2}$ In this review, when external help for patients and informal caregivers' existing problems was inadequate, any level of need for addressing the unsolved problems was regarded as unmet care needs, which included low, moderate, and high needs.

${ }^{3}$ In this paper, direct quotations from several included quantitative studies using commonly utilized research scales with documented psychometric properties were details of each of the used research questionnaire items. Thus, information regarding page numbers was not provided, but that for direct quotations from studies using self-designed semi-structured questionnaires and/or qualitative methods, as well as page numbers for such quotations, was provided.

\section{Abbreviations \\ 3LNQ: 3-Levels-of-Needs Questionnaire; ACIN: Advanced Cancer Information Needs; ADL: Activities of Daily Living; FIN: The Family Inventory of Needs; MMAT: Mixed Methods Appraisal Tool; NA-ACP: Needs Assessment of Advanced Cancer Patients; NAT: PD-C: Needs Assessment Tool: Progressive Disease-Cancer; PNAP: Patient Needs Assessment in Palliative Care; PNPC: Problems and Needs in Palliative Care questionnaire; PPUN: Caregiver's Perception of Patients' Unmet Needs; SCNS: Supportive Care Needs Survey; SCNS-P\&C: Partners and Caregivers supportive care needs survey; SNI: Spiritual Needs Inventory; TINQ-BC: Toronto Information Needs Questionnaire}

\section{Availability of data and materials}

Data that used for analysis in this review were all extracted from the original published studies (included studies) and were presented in Table 1 (Characteristics of the included studies).

\section{Authors' contributions}

Study conception and design, literature search, data extraction and checking, data synthesis and interpretation, and manuscript drafting and revision: TW; Study conception and design, and manuscript revision: AM and BPMC. Data extraction and checking, and manuscript revision: JYT. All authors read and approved the final manuscript.

\section{Ethics approval and consent to participate}

Not applicable

\section{Consent for publication}

Not applicable

\section{Competing interests}

The authors declare that there is no any conflict of interests regarding the publication of this paper.

\section{Publisher's Note}

Springer Nature remains neutral with regard to jurisdictional claims in published maps and institutional affiliations.

\section{Author details}

${ }^{1}$ School of Nursing, The Hong Kong Polytechnic University, Hung Hom, Hong Kong. ${ }^{2}$ College of Nursing and Midwifery, Charles Darwin University, Darwin, Australia.

Received: 26 May 2017 Accepted: 25 June 2018

Published online: 23 July 2018

\section{References}

1. World Health Organization. Global cancer rates could increase by $50 \%$ to 15 million by 2020. Available at: http://www.who.int/mediacentre/news/ releases/2003/pr27/en/. Last accessed 20 March 2017.

2. Thorne SE, Oliffe JL, Oglov V, Gelmon K. Communication challenges for chronic metastatic cancer in an era of novel therapeutics. Qual Health Res. 2013;7(23):863-75. https://doi.org/10.1177/1049732313483926.

3. Kim Y, Schulz R, Carver CS. Benefit finding in the cancer caregiving experience. Psychosom Med. 2007;69(3):283-91.

4. Gysels M, Higginson IJ, Rajasekaran M, et al. Improving supportive and palliative care for adults with cancer: research evidence. London: National Institute for Health and Clinical Excellence; 2004.

5. Okediji PT, Salako O, Fatiregun OO. Pattern and Predictors of Unmet Supportive Care Needs in Cancer Patients. Cureus. 2017;9(5):e1234.

6. Waller A, Girgis A, Johnson C, et al. Improving outcomes for people with progressive cancer: interrupted time series trial of a needs assessment intervention. J Pain Symptom Manag. 2012;43(3):569-81.

7. Moghaddam N, Coxon H, Nabarro S, et al. Unmet care needs in people living with advanced cancer: a systematic review. Support Care Cancer. 2016;24(8):3609-22.

8. Chen MC, Chen KM, Chu TP. Caregiver burden, health status, and learned resourcefulness of older caregivers. West J Nurs Res. 2015;37(6):767-80.

9. Cui J, Song $L J$, Zhou $L$, et al. Needs of family caregivers of advanced cancer patients: a survey in shanghai of China. Eur J Cancer Care. 2014;23(4):562-9.

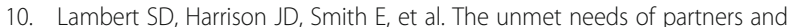
caregivers of adults diagnosed with cancer: a systematic review. BMJ Support Palliat Care. 2012;2(3):224-30

11. Grunfeld E, Coyle D, Whelan T, et al. Family caregiver burden: results of a longitudinal study of breast cancer patients and their principal caregivers. Can Med Assoc J. 2004;170(12):1795-801.

12. Proot IM, Abu-Saad $\mathrm{HH}$, Ter Meulen $\mathrm{RH}$, et al. The needs of terminally ill patients at home: directing one's life, health and things related to beloved others. Palliat Med. 2004;18(1):53-61.

13. Sharpe $L$, Butow $P$, Smith $C$, et al. The relationship between available support, unmet needs and caregiver burden in patients with advanced cancer and their carers. Psycho-Oncology. 2005:14(2):102-14.

14. Milbury K, Badr H, Fossella F, et al. Longitudinal associations between caregiver burden and patient and spouse distress in couples coping with lung cancer. Support Care Cancer. 2013;21(9):2371-9.

15. Hodgkinson K, Butow P, Hunt GE, et al. Life after cancer: couples' and partners' psychological adjustment and supportive care needs. Support Care Cancer. 2007;15(4):405-15.

16. World Health Organization. WHO definition of palliative care, 2012. Available at: http://www.who.int/cancer/palliative/definition/en/. Last Accessed 20 March 2017.

17. Wen KY, Gustafson DH. Needs assessment for cancer patients and their families. Health Qual Life Outcomes. 2004;2(1):11.

18. Valery PC, Powell E, Moses N, et al. Systematic review: unmet supportive care needs in people diagnosed with chronic liver disease. BMJ Open. 2015; 5(4):e007451.

19. Lam WW, Au AH, Wong JH, et al. Unmet supportive care needs: a crosscultural comparison between Hong Kong Chinese and German Caucasian women with breast cancer. Breast Cancer Res Treat. 2011;130(2):531-41.

20. Sanson-Fisher R, Girgis A, Boyes A, Bonevski B, Burton L, Cook P. The unmet supportive care needs of patients with cancer. Supportive Care Review Group. Cancer. 2000;88(1):226-37.

21. Harrison JD, Young JM, Price MA, et al. What are the unmet supportive care needs of people with cancer? A systematic review. Support Care Cancer. 2009;17(8):1117-28. 
22. Rainbird KJ, Perkins JJ, Sanson-Fisher RW. The needs assessment for advanced Cancer patients (NA-ACP): a measure of the perceived needs of patients with advanced, incurable cancer. A study of validity, reliability and acceptability. Psycho-Oncology. 2005;14(4):297-306.

23. Cancer Council. Living with advanced Cancer 2016. http://www.cancervic. org.au/about-cancer/advanced-cancer. Last accessed 30 Dec 2016.

24. Cancer Research UK. What is advanced bladder cancer? Available at: http:// www.cancerresearchuk.org/about-cancer/type/bladder-cancer/treatment/ advanced/what-is-advanced-bladder-cancer. Last accessed 30 Dec 2016.

25. Pace R, Pluye P, Gillian B, et al. Testing the reliability and efficiency of the pilot Mixed Methods Appraisal Tool (MMAT) for systematic mixed studies review. Int J Nurs Stud. 2012;49:47-53.

26. Hsieh HF, Shannon SE. Three approaches to qualitative content analysis. Qual Health Res. 2005;15(9):1277-88.

27. Ryan R; Cochrane Consumers and Communication Review Group. 'Cochrane Consumers and Communication Review Group: data synthesis and analysis'. http://cccrg.cochrane.org/author-resources, Last accessed 20 March 2017.

28. Morasso G, Capelli M, Viterbori P, et al. Psychological and symptom distress in terminal cancer patients with met and unmet needs. J Pain Symptom Manag. 1999;17(6):402-9.

29. Teunissen SC, de Haes HC, Voest EE, et al. Does age matter in palliative care? Crit Rev Oncol Hematol. 2006;60(2):152-8.

30. Osse BH, Vernooij-Dassen MJ, Schadé $\mathrm{E}$, et al. Problems experienced by the informal caregivers of cancer patients and their needs for support. Cancer Nurs. 2006;29(5):378-88.

31. Osse BH, Vernooij-Dassen MJ, Schadé $E$, et al. The problems experienced by patients with cancer and their needs for palliative care. Support Care Cancer. 2005;13(9):722-32.

32. Park SM, Kim YJ, Kim S, et al. Impact of caregivers' unmet needs for supportive care on quality of terminal cancer care delivered and caregiver's workforce performance. Support Care Cancer. 2010;18(6):699-706.

33. Hasegawa T, Goto N, Matsumoto N, et al. Prevalence of unmet needs and correlated factors in advanced-stage cancer patients receiving rehabilitation. Support Care Cancer. 2016;24(11):4761-7.

34. Uitdehaag MJ, Verschuur EM, van Eijck CH, et al. Problems and needs in patients with incurable esophageal and Pancreaticobiliary Cancer. Gastroenterol Nurs. 2015;38(1):42-54.

35. Chen SC, Chiou SC, Yu CJ, et al. The unmet supportive care needs-what advanced lung cancer patients' caregivers need and related factors. Support Care Cancer. 2016;24(7):2999-3009.

36. Effendy C, Vissers $\mathrm{K}$, Osse BH, et al. Comparison of problems and unmet needs of patients with advanced cancer in a European country and an Asian country. Pain Practice. 2015;15(5):433-40.

37. Vilalta A, Valls J, Porta J, et al. Evaluation of spiritual needs of patients with advanced cancer in a palliative care unit. J Palliat Med. 2014;17(5):592-600.

38. Schenker Y, Park SY, Maciasz R, et al. Do patients with advanced cancer and unmet palliative care needs have an interest in receiving palliative care services? J Palliat Med. 2014;17(6):667-72.

39. Fukui S. Information needs and the related variables of Japanese family caregivers of terminally ill cancer patients. Nurs Health Sci. 2004;6(1):29-36.

40. DuBenske LL, Wen KY, Gustafson DH, et al. Caregivers' differing needs across key experiences of the advanced cancer disease trajectory. Palliative and Supportive Care. 2008;6(03):265-72.

41. Uchida M, Akechi T, Okuyama T, et al. Patients' supportive care needs and psychological distress in advanced breast cancer patients in Japan. Jpn J Clin Oncol. 2011;41(4):530-6.

42. Liao YC, Liao WY, Shun SC, et al. Symptoms, psychological distress, and supportive care needs in lung cancer patients. Support Care Cancer. 2011; 19(11):1743-51.

43. Bužgová R, Hajnová E, Sikorová L, et al. Association between unmet needs and quality of life in hospitalised cancer patients no longer receiving anticancer treatment. Eur J Cancer Care. 2014;23(5):685-94.

44. Voogt $E$, van Leeuwen AF, Visser AP, et al. Information needs of patients with incurable cancer. Support Care Cancer. 2005;13(11):943-8.

45. Johnsen AT, Petersen MA, Pedersen $L$, et al. Do advanced cancer patients in Denmark receive the help they need? A nationally representative survey of the need related to 12 frequent symptoms/problems. Psycho-Oncology. 2013:22(8):1724-30.

46. Houts PS, Harvey HA, Hartz AJ, et al. Unmet needs of persons with cancer in Pennsylvania during the period of terminal care. Cancer. 1988;62(3):627-34
47. Khan L, Chiang A, Barnes E, et al. Needs assessment of patients and their caregivers at the rapid response radiotherapy program. J Pain Manag. 2012; 5(2):153-62

48. Dehghan R, Ramakrishnan J, Uddin-Ahmed N, et al. They patiently heard what we had to say... this felt different to me': the palliative care needs and care experiences of advanced cancer patients and their families in Bangladesh. BMJ Support Palliat Care. 2012;2(2):145-9.

49. Mangan PA, Taylor KL, Yabroff KR, et al. Caregiving near the end of life: unmet needs and potential solutions. Palliat Support Care. 2003;1 (03):247-59.

50. Fitch MI. Supportive care needs of patients with advanced disease undergoing radiotherapy for symptom control. Can Oncol Nurs J. 2012; 22(2):84-91.

51. Joad AS, Mayamol TC, Chaturvedi M. What does the informal caregiver of a terminally ill cancer patient need? A study from a cancer Centre. Ind J Palliat Care. 2011;17(3):191-6.

52. Buck HG, McMillan SC. The unmet spiritual needs of caregivers of patients with advanced cancer. J Hosp Palliat Nurs. 2008;10(2):91-9.

53. Deng D, Deng Q, Liu X, et al. Expectation in life review: a term of spiritual needs easily understood by Chinese hospice patients. Am J Hosp Palliat Care. 2015;32(7):725-31.

54. Rachakonda K, George M, Shafiei M, et al. Unmet supportive Cancer care needs: an exploratory quantitative study in rural Australia. World J Oncol. 2015;6(4):387-93.

55. Rainbird K, Perkins J, Sanson-Fisher R, et al. The needs of patients with advanced, incurable cancer. Br J Cancer. 2009;101(5):759-64.

56. Wong RK, Franssen E, Szumacher E, et al. What do patients living with advanced cancer and their carers want to know? -a needs assessment. Support Care Cancer. 2002;10(5):408-15.

57. Carter N, Bryant-Lukosius D, DiCenso A, et al. The supportive care needs of family members of men with advanced prostate cancer. Canadian oncology nursing journal/revue canadienne de soins infirmiers en. Oncologie. 2010; 20(4):166-70.

58. Au A, Lam W, Tsang J, et al. Supportive care needs in Hong Kong Chinese women confronting advanced breast cancer. Psycho-Oncology. 2013;22(5):1144-51.

59. Hwang SS, Chang VT, Alejandro Y, et al. Caregiver unmet needs, burden, and satisfaction in symptomatic advanced cancer patients at a veterans affairs (VA) medical center. Palliat Support Care. 2003;1(04):319-29.

60. Aranda S, Schofield P, Weih L, et al. Mapping the quality of life and unme needs of urban women with metastatic breast cancer. Eur J Cancer Care. 2005;14(3):211-22

61. Lelorain S, Brédart A, Dolbeault S, et al. How does a physician's accurate understanding of a cancer patient's unmet needs contribute to patient perception of physician empathy? Patient Educ Couns. 2015;98(6):734-41.

62. Lee HT, Melia KM, Yao CA, et al. Providing hospice home care to the terminally ill elderly people with cancer in Taiwan: family experiences and needs. Am J Hosp Palliat Med. 2014;31(6):628-35.

63. Liu Y. The status of the needs and service utilization wills of advanced cancer patients and their informal caregivers and service contents of some community health service centers delivery in Shanghai. 2008. The Second Military Medical University, Master Thesis. (in Chinese).

64. Chen $H Y$, Ju BB, Lu BQ, et al. Investigation and intervention of nursing demand and emotional state of family members of patients with advanced neoplasm. Shanghai Nurs. 2008;8(6):18-20.

65. Gu WJ, Shi YX, Yuan W, et al., Health service needs of terminal inpatients with advanced malignant tumor in community hospice care pilot settings in shanghai. Chinese General Practice 2015., 18 (22):2655-2661. (in Chinese).

66. Huang J, Xu Y, Peng P, et al. A survey of advanced cancer patients' community health service needs. Nurs J Chinese People's Liberation. 2008; 25(4A):33-5.

67. Miu J, Cao WQ, Wang XY, et al. Investigation on community care needs of patients with cancer and their family members in a community in shanghai. Chin Nurs Res. 2016;30(7A):2386-90.

68. Waller A, Girgis A, Johnson C, et al. Implications of a needs assessment intervention for people with progressive cancer: impact on clinical assessment, response and service utilisation. Psycho-Oncology. 2012; 21(5):550-7.

69. Templeton $\mathrm{H}$, Coates $\mathrm{V}$. Informational needs of men with prostate cancer on hormonal manipulation therapy. Patient Educ Couns. 2003;49(3):243-56.

70. Hwang SS, Chang VT, Cogswell J, et al. Study of unmet needs in symptomatic veterans with advanced cancer: incidence, independent 
predictors and unmet needs outcome model. J Pain Symptom Manag. 2004;28(5):421-32.

71. Murray SA, Kendall M, Boyd K, et al. Exploring the spiritual needs of people dying of lung cancer or heart failure: a prospective qualitative interview study of patients and their carers. Palliat Med. 2004;18(1):39-45.

72. Soelver $\mathrm{L}$, Rydahl-Hansen $\mathrm{S}$, Oestergaard B, et al. Identifying factors significant to continuity in basic palliative hospital care-from the perspective of patients with advanced cancer. J Psychosoc Oncol. 2014; 32(2):167-88.

73. Carter N, Bryant-Lukosius D, DiCenso A, et al. The supportive care needs of men with advanced prostate cancer. Oncol Nurs Forum. 2011;38(2):189-98.

74. Christ G, Siegel K. Monitoring quality-of-life needs of cancer patients. Cancer. 1990;65:760-5.

75. Lam WW, Tsang J, Yeo W, et al. The evolution of supportive care needs trajectories in women with advanced breast cancer during the 12 months following diagnosis. Support Care Cancer. 2014;22(3):635-44.

76. Deshields TL, Rihanek A, Potter P, et al. Psychological aspects of caregiving: perception of cancer patients and family caregivers. Support Care Cancer. 2012;20:349-56.

77. Gu X, Chen M, Liu M, et al. End-of-life decision-making of terminally ill cancer patients in a tertiary cancer center in shanghai, China. Support Care Cancer. 2016:24(5):2209-15.

78. Health Care Guideline- Palliative Care for Adults (Fifth Edition, November 2013). Available at: https://www.icsi.org/guidelines_more/catalog_ guidelines_and_more/catalog_guidelines/catalog_palliative_care_ guidelines/palliative_care/. Last accessed 1 Nov2016.

79. Hudson P, Remedios C, Zordan R, et al. Guidelines for the psychosocial and bereavement support of family caregivers of palliative care patients. J Palliat Med. 2012;15(6):696-702

80. Halkett GK, Lobb EA, Miller L, et al. Protocol for the care-IS trial: a randomised controlled trial of a supportive educational intervention for carers of patients with high-grade glioma (HGG). BMJ Open. 2015; 5(10):e009477.

81. Sealey M, Breen LJ, O'Connor M, et al. A scoping review of bereavement risk assessment measures: implications for palliative care. Palliat Med. 2015;29(7):577-89.

82. Grypdonck MH. Qualitative health research in the era of evidence-based practice. Qual Health Res. 2006;16(10):1371-85.

83. Britten N, Campbell R, Pope C, et al. Using meta ethnography to synthesise qualitative research: a worked example. J Health Serv Res Policy. 2002;7(4):209-15.

84. Field D, Clark D. Researching palliative care; 2001. p. 146-51.

\section{Ready to submit your research? Choose BMC and benefit from:}

- fast, convenient online submission

- thorough peer review by experienced researchers in your field

- rapid publication on acceptance

- support for research data, including large and complex data types

- gold Open Access which fosters wider collaboration and increased citations - maximum visibility for your research: over $100 \mathrm{M}$ website views per year 